\title{
Study of Rare Mesonic Decays Involving Di-Neutrinos in Their Final State
}

\author{
Azeem Mir, ${ }^{1}$ Farida Tahir, ${ }^{1}$ Shakeel Mahmood $\mathbb{D}^{2},{ }^{2}$ and Shi- Hai Dong $\mathbb{C}^{3}$ \\ ${ }^{1}$ Physics Department, COMSATS Institute of Information Technology, Islamabad, Pakistan \\ ${ }^{2}$ Air University, PAF Complex, Service Road, E-9, Islamabad, Pakistan \\ ${ }^{3}$ CIDETEC, Instituto Politécnico Nacional, Unidad Profesional, Adolfo López Mateos, CDMX 07700, Mexico
}

Correspondence should be addressed to Shakeel Mahmood; shakeel_mahmood@hotmail.com

Received 1 December 2017; Revised 15 March 2018; Accepted 11 April 2018; Published 1 August 2018

Academic Editor: Adrian Buzatu

Copyright (C) 2018 Azeem Mir et al. This is an open access article distributed under the Creative Commons Attribution License, which permits unrestricted use, distribution, and reproduction in any medium, provided the original work is properly cited. The publication of this article was funded by SCOAP

\begin{abstract}
We have studied phenomenological implication of R-parity violating $\left(\not R_{p}\right)$ Minimal Supersymmetric Model (MSSM) via analyses of pure leptonic $(M \longrightarrow \nu \bar{\nu})$ and semileptonic decays of pseudoscalar mesons $(M \longrightarrow X \nu \bar{\nu})$. These analyses involve comparison between theoretical predictions made by $\not k_{p}$ MSSM and the Standard Model (SM) with the experimental results like branching fractions $(B r)$ of the said process. We have found, in general, that $\not R_{p}$ contribution dominates over the SM contribution, i.e., by a factor of 10 for the pure leptonic decays of $K_{L, S}$ and by $10^{2}$ and $10^{4}$ in case of $B_{s}$ and $B_{d}$, respectively. Furthermore, the limits obtained on $\not \chi_{p}$ Yukawa couplings $\left(\lambda_{\alpha k \beta}^{\prime} \lambda_{\alpha k \gamma}^{\prime *}\right)$ by using $B r(M \longrightarrow X \nu \bar{v})$ are used to calculate $B r(M \longrightarrow \nu \bar{\nu})$. This demonstrates the role of $\not R_{p}$ MSSM as a viable model for the study of new physics contribution in rare decays at places like Super B factories, KOTO (J-PARC) and NA62 at CERN.
\end{abstract}

\section{Introduction}

Flavor Changing Neutral Currents (FCNC) that mediate different flavored fermions (quarks) of the same charge are one of the most important tools searching for physics beyond the Standard Model (SM). This is due to their rarity owing to the GIM mechanism [1]. FCNC processes involving leptons are strictly forbidden in SM due to lepton family number conservation contrary to established experimental facts [213], and such processes can only be accommodated through physics beyond the SM. However, lepton flavor conserving processes can proceed through both universal and nonuniversal weak neutral current interactions. Here, universal weak neutral current interactions correspond to the SM interactions, which are flavor as well as generation blind, and non-universal weak neutral current interactions represent new physics (NP) interactions which are flavor as well as generation sensitive. Analyses, involving the bounds on NP couplings, of such type of processes are good for comparative study of different models. In this paper, we have presented one class of such type of pure leptonic and semileptonic decays of pseudoscalar mesons involving di-neutrinos in their final state in the framework of SM and R-parity violating $\left(R_{p}\right)$ supersymmetric (SUSY) model.

Leptonic and semileptonic decays of beauty and strange mesons have played an important role in measuring parameters related to Cabibbo-Kobayashi-Maskawa (CKM), unitary angles, and also in probing CP-violation [14-16]. Many NP models like 2HDM [17] and $R_{p}$ Minimal Supersymmetric Standard Model (MSSM) [18-20] have been explored in these processes [21-33] as well. Super B factories [34, 35] and experimental set-ups like KOTO at J-PARC and NA62 at CERN [36-39] hold a lot of potential in this regard. $\mathrm{LHCb}$ also holds a lot of promise for discovering prospects of NP in B decays $[40,41]$.

MSSM [42-47] is the most economical version of SUSY. It is also the minimal extension of SM [42-47]. MSSM allows processes that violate baryon and lepton number. It also allows Lepton flavor violating (LFV) processes (that do violate lepton family number). R-parity, a discrete symmetry, is 
imposed to prevent baryon number, lepton number, and flavor violating processes. It is defined as $R_{p}=(-1)^{3 B+L+2 S}[42$, $48,49]$. R-parity conservation is phenomenologically motivated and if relaxed carefully allows one to analyze rare and forbidden decays while maintaining the stability of matter [46, 50-52]. The R-parity violating gauge invariant and renormalizable superpotential is [42, 48, 49]

$$
\begin{aligned}
W_{\not k_{p}}= & \frac{1}{2} \lambda_{i j k} L_{i} L_{j} E_{k}^{c}+\lambda_{i j k}^{\prime} L_{i} Q_{j} D_{k}^{c}+\frac{1}{2} \lambda_{i j k}^{\prime \prime} U_{i}^{C} D_{j}^{C} D_{k}^{C} \\
& +\mu_{i} H_{u} L_{i},
\end{aligned}
$$

where $i, j, k$ are generation indices, $L_{i}$ and $Q_{i}$ are the lepton and quark left-handed $S U(2)_{L}$ doublets, and $E^{c}, D^{c}$ are the charge conjugates of the right-handed leptons and quark singlets, respectively. Here $\lambda_{i j k}, \lambda_{i j k}^{\prime}$, and $\lambda_{i j k}^{\prime \prime}$ are the Yukawa couplings. The term proportional to $\lambda_{i j k}$ is antisymmetric in first two indices $[i, j]$ and $\lambda_{i j k}^{\prime \prime}$ is antisymmetric in last two indices $[j, k]$, implying $9\left(\lambda_{i j k}\right)+27\left(\lambda_{i j k}^{\prime}\right)+9\left(\lambda_{i j k}^{\prime \prime}\right)=45$ independent coupling constants among which 36 are related to the lepton flavor violation (9 from $L L E^{\mathcal{C}}$ and 27 from $\left.L Q D^{c}\right)$. We can rotate the last term away without affecting things of our interest.

In this scenario for detailed illustration we will use the pure and semileptonic rare decays of pseudoscalar mesons with neutrinos in the final state, i.e., $M^{0} \longrightarrow v_{\alpha} \bar{\nu}_{\beta}, M^{ \pm, 0} \longrightarrow$ $N^{ \pm, 0} v_{\alpha} \bar{\nu}_{\beta}$, and $M^{ \pm, 0} \longrightarrow N^{ \pm, 0} v_{\alpha} \bar{\nu}_{\beta}$, where $M=K, B$ and $N=\pi, K$. At the quark level, all $M^{ \pm, 0} \longrightarrow N^{ \pm, 0} v_{\alpha} \bar{\nu}_{\beta}$ decays are represented by $s(b) \longrightarrow d(d, s) q v_{\alpha} \bar{v}_{\beta}(q=d, s)$ and (all these processes can be) divided into two categories on the bases of lepton flavors, i.e.,

(1) lepton flavor conserving $(\alpha=\beta)$,

(2) lepton flavor violating $(\alpha \neq \beta)$ decays.

The first type of decays $s(b) \longrightarrow d(d, s) \nu_{\alpha} \bar{\nu}_{\alpha}(\alpha=e, \mu, \tau)$ is absent in the SM at tree level and is however induced by GIM mechanism [1] at the quantum loop level [53] which makes their effective strength very small, further suppression caused by the CKM matrix $[54,55]$. These two suppressions make FCNC decays very rare. Furthermore, these processes will provide indirect test of high energy scales through a low energy process. Such type of processes has only short distance dominant contribution whereas long distance contribution is subleading [56], as we are taking pure and semileptonic decays, which can be accurately predicted in the SM due to the fact that the only relevant hadronic operators are just the current operators whose matrix elements can be extracted from their respective leading decays [57-61].

The second type of decays $s(b) \longrightarrow d(d, s) v_{\alpha} \bar{v}_{\beta}(\alpha \neq$ $\beta ; \alpha, \beta=e, \mu, \tau)$ is strictly forbidden to all orders in the SM due to lepton flavor violation, so their detection can clearly signal the presence of new interactions. Hence one can say that these are the "golden channels" for the study of NP.

In this paper, we have analyzed the above-mentioned decays in the SM (first case) and then in $\not_{p}$ violating MSSM. Our focus is to compare the NP contribution to the branching fraction of decay processes (under consideration) with the
SM prediction and also with the experimental limits. In the forthcoming section, we will discuss these processes one by one.

2. $s \longrightarrow d \nu_{\alpha} \bar{\nu}_{\alpha}$

In the SM, the effective Hamiltonian for the semileptonic $\left(K \longrightarrow \pi v_{\alpha} \bar{\nu}_{\alpha}, K \longrightarrow \pi^{0} \nu_{\alpha} \bar{\nu}_{\alpha}\right)$ and pure leptonic $K_{L, S} \longrightarrow$ $\nu_{\alpha} \bar{\nu}_{\alpha}$ processes is given by $[62,63]$

$$
H_{e f f}=\sum_{l} C_{S M}(\bar{s} d)_{V-A}\left(\bar{\nu}_{l} \nu_{l}\right)_{V-A} .
$$

In this case, all leptons couple universally with the electroweak gauge bosons, where

$$
\begin{aligned}
C_{S M} & =\frac{G_{F} \alpha}{2 \sqrt{2} \sin ^{2} \theta_{w}}\left(V_{c s}^{*} V_{c d} X_{N L}^{l}+V_{t s}^{*} V_{t d} X\left(x_{t}\right)\right), \\
X\left(x_{t}\right) & =X_{0}\left(x_{t}\right)+\frac{\alpha_{S}}{4 \pi} X_{l}\left(x_{t}\right),
\end{aligned}
$$

where $X_{0}\left(x_{t}\right)=\left(x_{t} / 8\right)\left(\left(x_{t}+2\right) /\left(x_{t}-1\right)+\left(\left(3 x_{t}-6\right) /\left(x_{t}-\right.\right.\right.$ $\left.\left.1)^{2}\right) \log x_{t}\right)$ and

$$
\begin{aligned}
& X_{l}\left(x_{t}\right) \\
& =\frac{-23 x_{t}+5 x_{t}^{2}-4 x_{t}^{3}}{3\left(1-x_{t}\right)^{2}}+\frac{x_{t}-11 x_{t}^{2}+x_{t}^{3}+x_{t}^{4}}{\left(1-x_{t}\right)^{3}} \log x_{t} \\
& +\frac{8 x_{t}+4 x_{t}^{2}+x_{t}^{3}-x_{t}^{4}}{2\left(1-x_{t}\right)^{3}}\left(\log x_{t}\right)^{2} \\
& -\frac{4 x_{t}-x_{t}^{3}}{\left(1-x_{t}\right)^{2}} \int_{1}^{x_{t}} d t \frac{\log t}{1-t}+\gamma_{m}^{(0)} x_{t} \frac{\partial X_{0}\left(x_{t}\right)}{\partial x_{t}} \log \frac{\mu_{t}^{2}}{M_{W}^{2}}, \\
& X_{N L}^{l}=C_{N L}-4 B_{N L}^{(1 / 2)} \text {, } \\
& \text { and } x_{t}=\bar{m}_{t}^{2}\left(\mu_{t}\right) / M_{W}^{2}, \mu_{t}=O\left(m_{t}\right), C_{N L} \text { and } B_{N L}^{(1 / 2)} \text { are } \\
& L_{\not k_{\mathrm{p}}}^{e f f}\left(s \longrightarrow d+v_{\alpha}+\bar{v}_{\alpha}\right) \\
& =\frac{4 G_{F}}{\sqrt{2}}\left[A_{\alpha \beta}^{s d}\left(\bar{v}_{\alpha} \gamma^{\mu} P_{L} \nu_{\alpha}\right)\left(\bar{d} \gamma_{\mu} P_{R} s\right)\right],
\end{aligned}
$$

where $\alpha=e, \mu$. The first term in (2) comes from the down squark exchange (where $d$ and $s$ are down type quarks). The dimensionless coupling constant $A_{\alpha \alpha}^{s d}$ is related to $\not_{p}$ Yukawa couplings $\lambda_{\alpha k 1}^{\prime} \lambda_{\alpha k 2}^{\prime *}$ by

$$
A_{\alpha \alpha}^{s d}=\frac{\sqrt{2}}{4 G_{F}} \sum_{k=1}^{3} \frac{\lambda_{\alpha k 1}^{\prime} \lambda_{\alpha k 2}^{\prime *}}{2 m_{\widetilde{d_{k}^{c}}}^{2}} .
$$


The differential decay rate $d \Gamma / d q^{2}$ for the semileptonic decay processes is given by [62-64]

$$
\frac{d \Gamma}{d q^{2}}=\frac{1}{2^{5} \pi^{5}} \lambda^{3 / 2}\left(1, r_{M}, s\right) m_{K}^{3}\left|f_{p}^{+}\left(q^{2}\right)\right|^{2}\left|C_{l}\right|^{2},
$$

where $\lambda\left(1, r_{M}, s\right)=1+r_{M}^{2}+s^{2}-2 r_{M}-2 s-2 r_{M} s$ with $r_{M}=$ $\left(m_{M} / m_{B}\right)^{2}$ and $s=q^{2} / m_{B}^{2}, C_{l}=C_{S M}+z(1 / 8) e^{i \theta} ; z$ is the general NP parameter. We have used the value for the form factor $f_{p}^{+}\left(q^{2}\right)$ for the above decay processes of $K^{+}$and $K^{0}$ as given in [65]. Since this work focuses on $\not_{p}$ MSSM, we will shift our focus to $z$ (for the calculation of limits on couplings) and Yukawa couplings $\lambda_{\alpha k 1}^{\prime} \lambda_{\alpha k 2}^{\prime *} / m_{\widetilde{d_{k}^{c}}}^{2}$ (for the predictions of branching fraction). The decay rate for pure leptonic decay processes is given by

$$
\Gamma\left(s \longrightarrow d v_{l} \bar{\nu}_{l}\right)=\frac{1}{8 \pi} m_{K}^{3}\left|f_{p}\left(q^{2}\right)\right|^{2}\left|\frac{2 m_{l}}{m_{K}} C_{l}\right|^{2} .
$$

The form factor $f_{p}\left(q^{2}\right)$ is given by [66]. $m_{K}$ represents the mass of strange meson and $m_{l}$ is the mass of lepton, where $C_{l}$ is same as that of semileptonic decays.

$$
\text { 3. } b \longrightarrow d(s) v_{\alpha} \nu_{\alpha}
$$

In MSSM, the relevant effective Lagrangian for the decay process $B \longrightarrow \pi(K) \nu_{\alpha} \bar{\nu}_{\alpha}$ is given by [57-61]

$$
\begin{aligned}
& L_{\not k_{\mathrm{p}}}^{\text {eff }}\left(b \longrightarrow d(s)+v_{\alpha}+\bar{\nu}_{\alpha}\right) \\
& \quad=\frac{4 G_{F}}{\sqrt{2}}\left[A_{\alpha \alpha}^{b d(s)}\left(\bar{\nu}_{\alpha} \gamma^{\mu} P_{L} \nu_{\alpha}\right)\left(\bar{b} \gamma_{\mu} P_{R} d(s)\right)\right],
\end{aligned}
$$

where $\alpha=e, \mu$. The first term in (2) comes from the down squark exchange (where $b$ and $d(s)$ are down type quarks). The dimensionless coupling constant $A_{\alpha \alpha}^{b d(s)}$ is given by

$$
A_{\alpha \alpha}^{b d(s)}=\frac{\sqrt{2}}{4 G_{F}} \sum_{k=1}^{3} \frac{\lambda_{\alpha k 1(2)}^{\prime} \lambda_{\alpha k 3}^{\prime *}}{2 m_{\bar{d}_{k}^{c}}^{2}} .
$$

The differential decay rate for semileptonic decay processes is given by [62-64]

$$
\frac{d \Gamma}{d q^{2}}=\frac{1}{2^{5} \pi^{5}} \lambda^{3 / 2}(1, r, s) m_{B}^{3}\left|f_{p}^{+}\left(q^{2}\right)\right|^{2}\left|C_{l}\right|^{2},
$$

where

$$
C_{l}=C_{S M}+\frac{z}{8} e^{i \theta},
$$

with

$$
C_{S M}=\frac{G_{F} \alpha}{2 \sqrt{2} \sin ^{2} \theta_{w}}\left(V_{c b}^{*} V_{c d(s)} X_{N L}^{l}+V_{t b}^{*} V_{t d(s)} X\left(x_{t}\right)\right),
$$

with

$$
\begin{aligned}
X\left(x_{t}\right) & =X_{0}\left(x_{t}\right)+\frac{\alpha_{S}}{4 \pi} X_{l}\left(x_{t}\right), \\
x_{t} & =\frac{\bar{m}_{t}^{2}\left(\mu_{t}\right)}{M_{W}^{2}}, \\
\mu_{t} & =O\left(m_{t}\right),
\end{aligned}
$$

and $z$ as explained in the above section is the general NP parameter and $\lambda_{\alpha k 1(2)}^{\prime} \lambda_{\alpha k 3}^{\prime *} / m_{\overrightarrow{d_{k}^{c}}}^{2}$. We have used the form factor $f_{p}^{+}\left(q^{2}\right)$ for the above decay processes of $B->\pi(K)$ as given in [67]. The decay rate for pure leptonic decay processes is given by $[46,50-52]$

$$
\Gamma\left(b \longrightarrow d(s) v_{l} \bar{v}_{l}\right)=\frac{1}{8 \pi} m_{B}^{3}\left|f_{p}\left(q^{2}\right)\right|^{2}\left|\frac{2 m_{l}}{m_{B}} C_{l}\right|^{2} .
$$

The form factor $f_{p}\left(q^{2}\right)$ is given by [66], $m_{B}$ represents the mass of beauty meson and $m_{l}$ is the mass of lepton.

\section{Results and Discussions}

We have carried out study of hypercharge changing two and three body decay processes of pseudoscalar mesons $(M \longrightarrow$ $X \nu_{\alpha} \bar{\nu}_{\alpha} ; M \longrightarrow v_{\alpha} \bar{\nu}_{\alpha}$ ), where $M=K, B ; \alpha=e, \mu, \tau$ and $X=$ $\pi, K$. This study considers two types of processes: polarized and unpolarized flavor of the lepton. The analysis carried out involves comparison of branching fraction of a certain decay process (mentioned above) calculated from both theoretical and experimental ground. This comparison not only helps to place bounds (listed in Tables 2, 4, and 5) on $R_{p}$ Yukawa couplings $\lambda_{\alpha k \beta}^{\prime} \lambda_{\alpha k \gamma}^{\prime *}$ but also enables to predict (listed in Tables 3 , 6-9) the enhancement of similar processes (having identical FCNC). The enhancement is given in three forms, namely, NP (contribution from Yukawa couplings only), Interference (product of $\mathrm{SM}$ contribution, coming from $C_{S M}$ and Yukawa couplings), and combined (NP+Interference). All the results are displayed in graphs plotted in Figures 2-13, which are composed of simple (variation of branching fraction with respect to the magnitude of NP parameter, i.e., $\left.\left|z\left(\lambda_{\rho \omega \sigma}^{\prime} \lambda_{\alpha \beta \gamma}^{\prime *}\right)\right|\right)$ and contour plot (region plot of magnitude and phase of NP parameter at different values of branching fraction within limits of experimental measurements). A visual error analysis for the experimental measurement of branching fraction is also presented in these graphs by constraining lines at mean and $\pm 1 \sigma$ level. Similar error analysis is repeated in tables. The Feynman diagrams and table listing experimental data [68] related to these processes are given in Figure 1 and Table 1 , respectively. The Yukawa couplings $\left(\lambda_{\rho \omega \sigma}^{\prime} \lambda_{\alpha \beta \gamma}^{\prime *}\right)$ involved are normalized to the square of $m_{d^{c}} / 100 \mathrm{GeV}$ in all these tables and figures.

First, we will discuss the results related to semileptonic decay processes followed by pure leptonic decays. We have plotted graphs in Figures 2 and 3 for the study of process $K \longrightarrow \pi v_{\alpha} \bar{v}_{a}$. These plots relate the branching fraction of the said process with the magnitude and phase of NP parameters $\left(\left|z\left(\lambda_{i k 1}^{\prime} \lambda_{i k 2}^{\prime *}\right)\right|\right.$ and $\left.\theta\right)$. Contour plots in Figure 2 represent the allowed region for NP parameters (magnitude and phase) for specific values of branching fraction. All four plots (comprising unpolarized (a) and polarized (b-d)) show that the maximum magnitude of NP parameter oscillates with respect to its phase in general. The plot in Figure 2(a) shows a particular pattern at given error, i.e., at $-1 \sigma$ level of measured branching fraction $\left(0.7 \times 10^{-7}\right)$. It clearly shows that only a narrow range of phase of NP parameter $(|\theta| \leq \pi / 4)$ is allowed for given $-1 \sigma$ level. The bounds on the magnitude 
TABLE 1: Table listing the properties of processes under discussion [68]. Here $|z|$ is the strength of the NP parameter.

\begin{tabular}{|c|c|c|c|c|}
\hline \multirow[t]{2}{*}{ Process } & \multirow[t]{2}{*}{ Experimental Measurement } & \multirow[t]{2}{*}{ SM Prediction } & \multicolumn{2}{|c|}{ Bounds on New Physics Parameter } \\
\hline & & & Magnitude $|z|$ & Phase $(\theta)$ \\
\hline$B \longrightarrow \pi v \bar{\nu}$ & $<9.8 \times 10^{-5}$ & $1.62 \times 10^{-7}$ & $<1.79 \times 10^{-6}$ & $\leq 2 \pi$ \\
\hline \multirow{3}{*}{$B_{d}^{0} \longrightarrow \nu \bar{v}$} & & $6.13 \times 10^{-31}(e)$ & & \\
\hline & & $4.57 \times 10^{-27}(\mu)$ & & $\leq 2 \pi$ \\
\hline & & $5.91 \times 10^{-25}(\tau)$ & & \\
\hline$B \longrightarrow K \nu \bar{\nu}$ & $<1.6 \times 10^{-5}$ & $4.40 \times 10^{-4}$ & $<8.69 \times 10^{-7}$ & $\leq 2 \pi$ \\
\hline \multirow{3}{*}{$B_{s}^{0} \longrightarrow v \bar{v}$} & & $1.36 \times 10^{-29}(e)$ & & \\
\hline & & $1.02 \times 10^{-25}(\mu)$ & & $\leq 2 \pi$ \\
\hline & & $1.32 \times 10^{-23}(\tau)$ & & \\
\hline \multirow{3}{*}{$K^{ \pm} \longrightarrow \pi \nu \bar{\nu}$} & & & $(7.83 \pm 2.01) \times 10^{-9}(e)$ & \\
\hline & $<(1.7 \pm 1.0) \times 10^{-5}$ & $8.23 \times 10^{-11}$ & $(7.83 \pm 2.01) \times 10^{-9}(\mu)$ & $\leq 2 \pi$ \\
\hline & & & $(7.81 \pm 2.01) \times 10^{-9}(\tau)$ & \\
\hline \multirow{3}{*}{$K_{s}^{0} \longrightarrow \nu \bar{\nu}$} & & $7.24 \times 10^{-33}(e)$ & & \\
\hline & & $5.40 \times 10^{-29}(\mu)$ & & $\leq 2 \pi$ \\
\hline & & $6.98 \times 10^{-27}(\tau)$ & & \\
\hline \multirow{3}{*}{$K_{L}^{0} \longrightarrow \nu \bar{v}$} & & $4.27 \times 10^{-33}(e)$ & & \\
\hline & & $3.19 \times 10^{-29}(\mu)$ & & $\leq 2 \pi$ \\
\hline & & $4.06 \times 10^{-27}(\tau)$ & & \\
\hline \multirow{6}{*}{$\begin{array}{l}K^{0} \longrightarrow \pi^{0} \nu \bar{\nu} \\
\text { (Bounds on New } \\
\text { Physics parameters } \\
\text { have been calculated } \\
\text { numerically for their } \\
\text { fit with SM } \\
\text { prediction) }\end{array}$} & & & $<1.9 \times 10^{-9}$ & $15^{\circ}$ \\
\hline & & & $<9.83 \times 10^{-10}$ & $30^{\circ}$ \\
\hline & $<2.6 \times 10^{-5}$ & $2.76 \times 10^{-11}$ & $<6.96 \times 10^{-10}$ & $45^{\circ}$ \\
\hline & & & $<5.68 \times 10^{-10}$ & $60^{\circ}$ \\
\hline & & & $<5.09 \times 10^{-10}$ & $75^{\circ}$ \\
\hline & & & $<4.92 \times 10^{-10}$ & $90^{\circ}$ \\
\hline
\end{tabular}

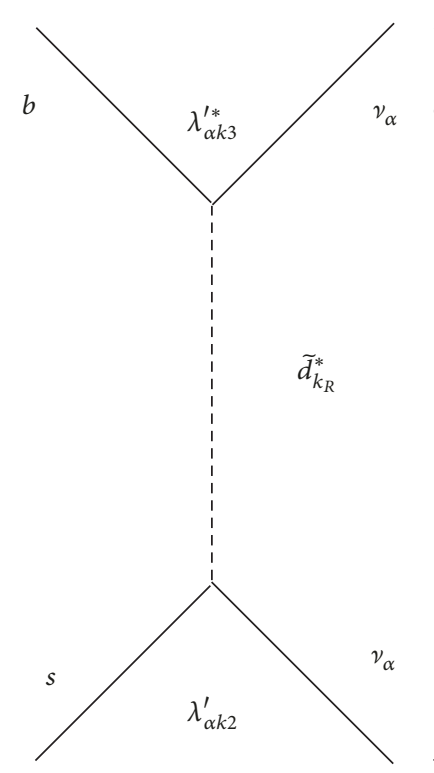

(a)

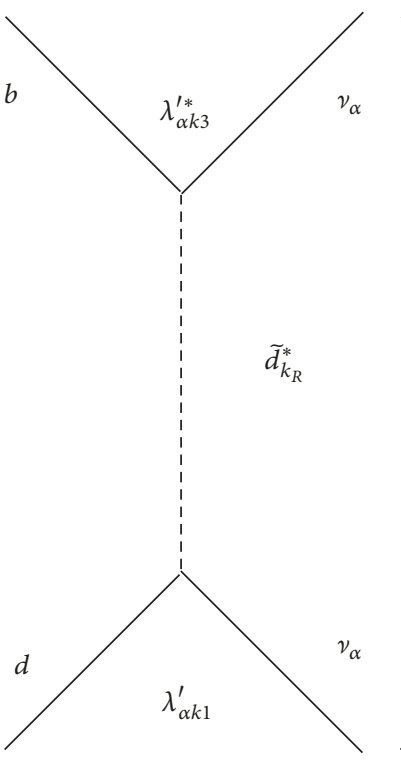

(b)

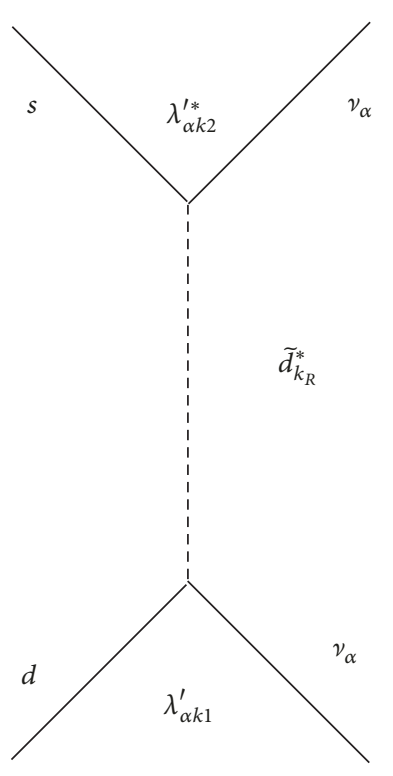

(c)

FIGURE 1: Feynman diagrams of (a) $b \longrightarrow s \nu_{\alpha} \bar{\nu}_{\alpha}$, (b) $b \longrightarrow d \nu_{\alpha} \bar{\nu}_{\alpha}$, (c) $s \longrightarrow d \nu_{\alpha} \bar{\nu}_{\alpha} . \alpha=1,2,3$. 


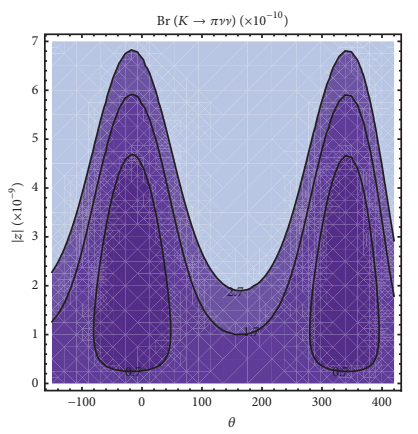

(a)

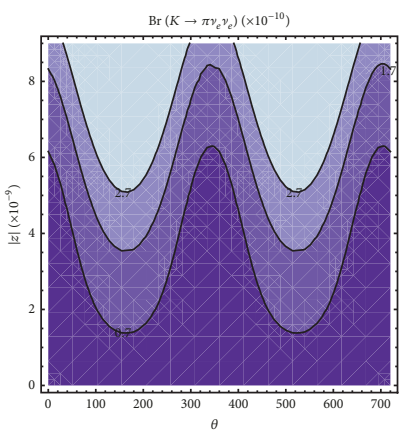

(b)

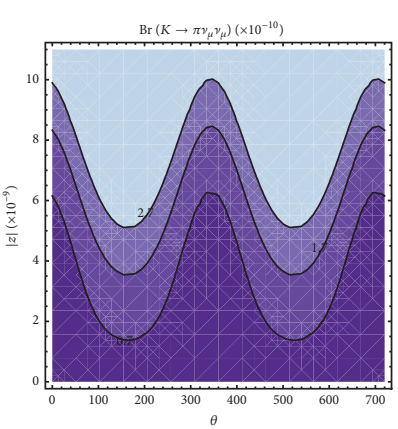

(c)

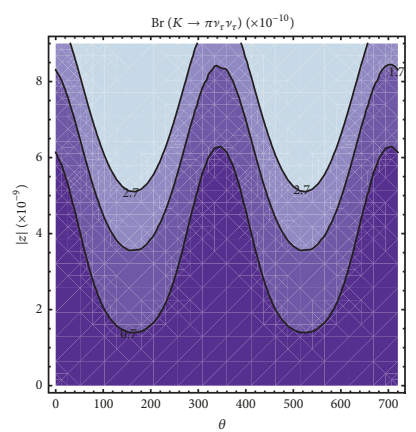

(d)

FIGURE 2: Allowed region of general NP parameters $(z, \theta)$ for $K \longrightarrow \pi v_{\alpha} \bar{\nu}_{\alpha}$ at several values of branching fraction. $\alpha$ is (a) unpolarized, (b) e, (c) $\mu$, (d) $\tau$. The three contours belong to branching fraction at $[0.7,1.7,2.7] \times 10^{-10}$.

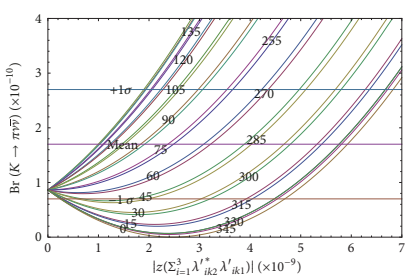

(a)

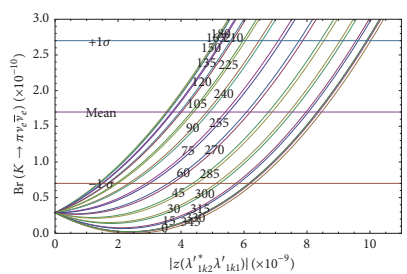

(b)

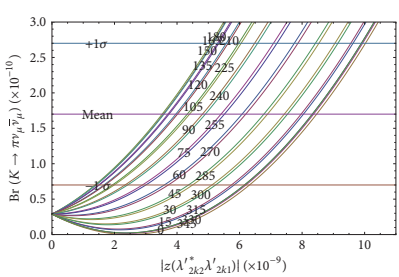

(c)

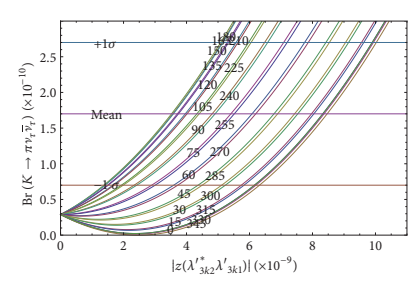

(d)

FIGURE 3: Variations of branching fraction w.r.t NP parameter $\left|z\left(\lambda_{i j \mathrm{k}}^{\prime} \lambda_{l \mathrm{mn}}^{\prime}\right)\right|$ at several values of $\theta$ for $K \longrightarrow \pi v_{\alpha} \bar{\nu}_{\alpha} . \alpha$ is (a) unpolarized, (b) $\mathrm{e},(\mathrm{c}) \mu,(\mathrm{d}) \tau$. The three bounds belong to branching fraction at $[0.7(-1 \sigma), 1.7($ Mean $), 2.7(+1 \sigma)] \times 10^{-10}$ corresponding to an experimental measurement of $(1.7 \pm 0.7) \times 10^{-10}$

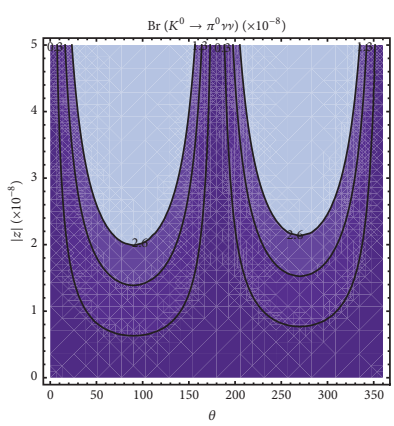

(a)

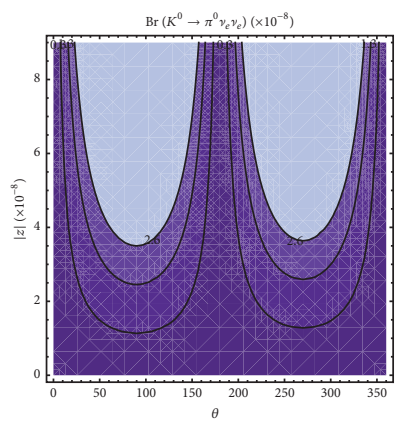

(b)

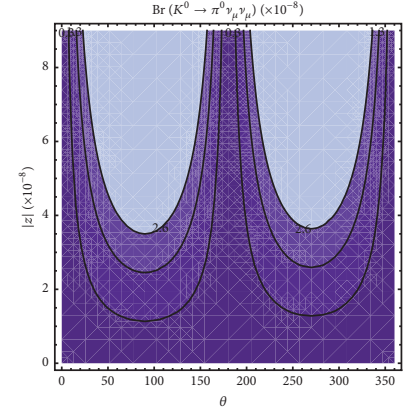

(c)

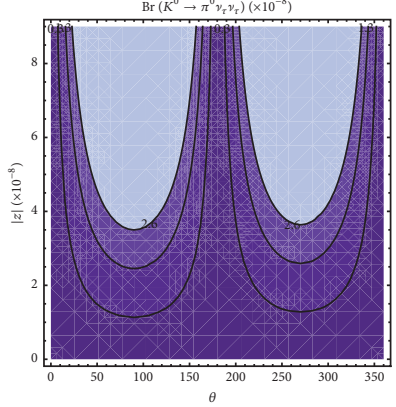

(d)

FIGURE 4: Allowed regions of general NP parameters $(z, \theta)$ for $K^{0} \longrightarrow \pi^{0} v_{\alpha} \bar{\nu}_{\alpha}$ at specific values of branching fraction. $\alpha$ is (a) unpolarized, (b) e, (c) $\mu$, (d) $\tau$. The three contours belong to branching fraction at $[0.3,1.3,2.6] \times 10^{-8}$.

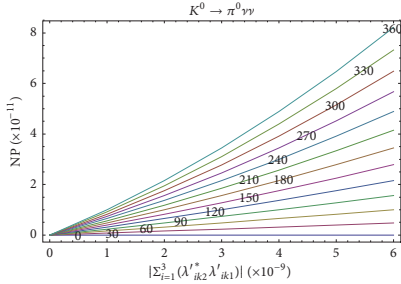

(a)

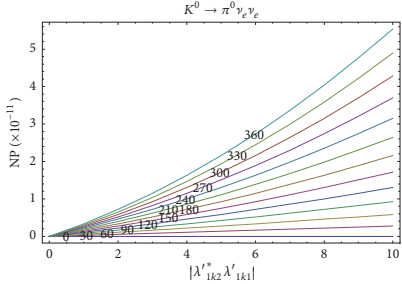

(b)

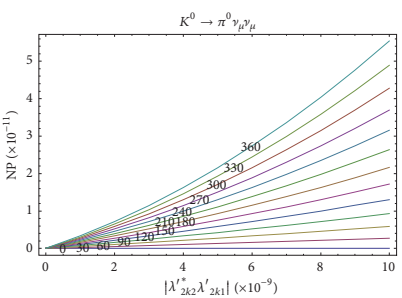

(c)

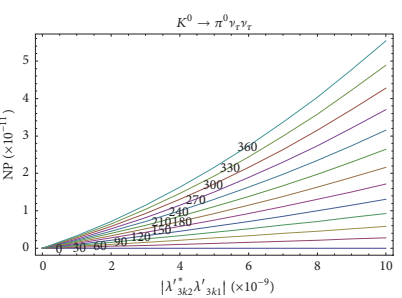

(d)

FIGURE 5: Variations of branching fraction (NP contribution only) with respect to NP parameter $\left|\lambda_{i j \mathrm{k}}^{\prime} \lambda_{l \mathrm{mn}}^{\prime}\right|$ at several values of $\theta$ for $K^{0} \longrightarrow$ $\pi^{0} \nu_{\alpha} \bar{\nu}_{\alpha} . \alpha$ is (a) unpolarized, (b) e, (c) $\mu$, (d) $\tau$. 


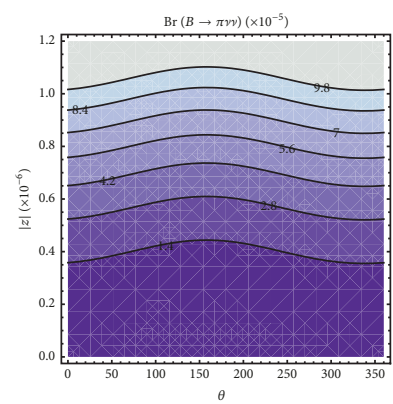

(a)

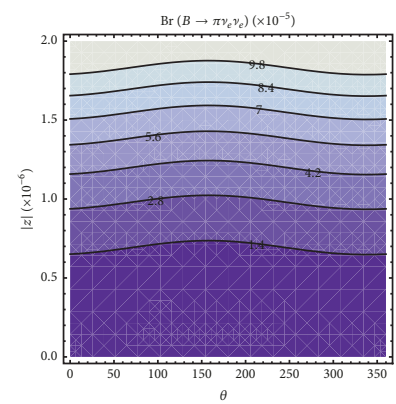

(b)

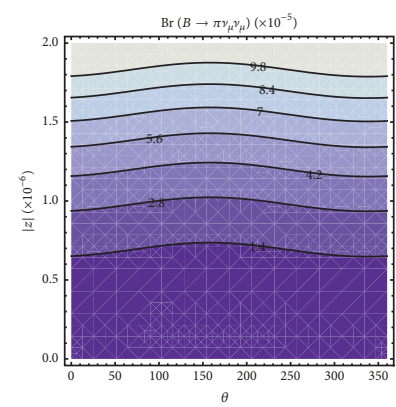

(c)

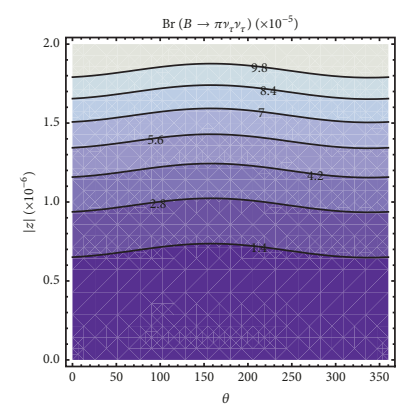

(d)

FIGURE 6: Allowed regions of general NP parameters $(z, \theta)$ for $B \longrightarrow \pi v_{\alpha} \bar{\nu}_{\alpha}$ at specific values of branching fraction. $\alpha$ is (a) unpolarized, (b) $\mathrm{e},(\mathrm{c}) \mu$, (d) $\tau$. The three contours belong to branching fraction at $[1.4-9.8] \times 10^{-6}$.

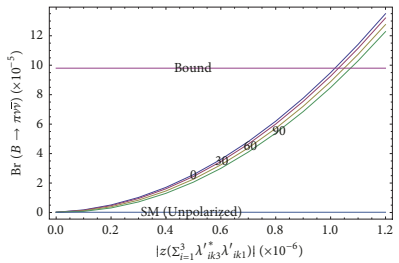

(a)

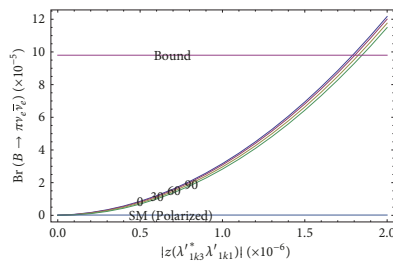

(b)

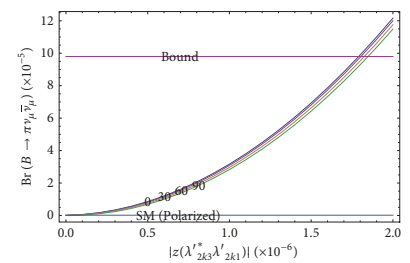

(c)

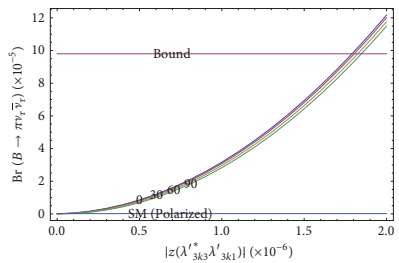

(d)

FIGURE 7: Variation of branching fraction with respect to NP parameter $\left|z\left(\lambda_{i j \mathrm{k}}^{\prime} \lambda_{l \mathrm{mn}}^{\prime}\right)\right|$ at several values of $\theta$ for $B \longrightarrow \pi v_{\alpha} \bar{\nu}_{\alpha} . \alpha$ is (a) unpolarized, (b) e, (c) $\mu$, (d) $\tau$. Experimental bound on the process is $9.8 \times 10^{-5}$.

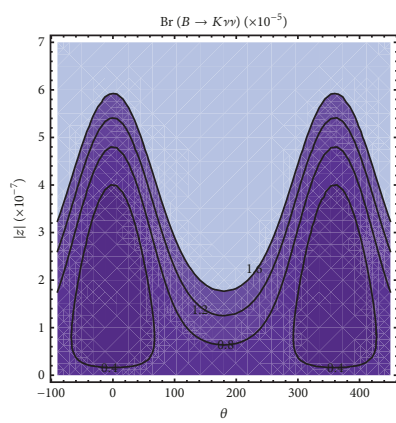

(a)

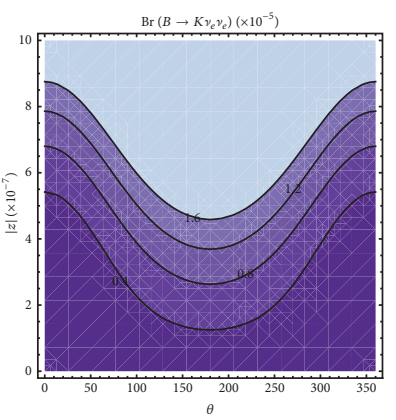

(b)

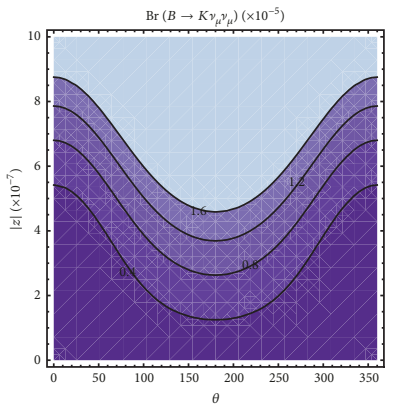

(c)

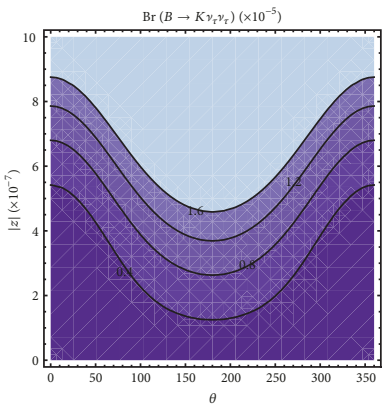

(d)

FIGURE 8: Allowed regions of general NP parameters $(z, \theta)$ for $B \longrightarrow K \nu_{\alpha} \bar{\nu}_{\alpha}$ at specific values of branching fraction. $\alpha$ is (a) unpolarized, (b) e, (c) $\mu$, (d) $\tau$. The three contours belong to branching fraction at $[0.4-1.6] \times 10^{-5}$.

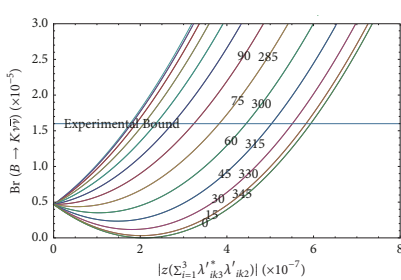

(a)

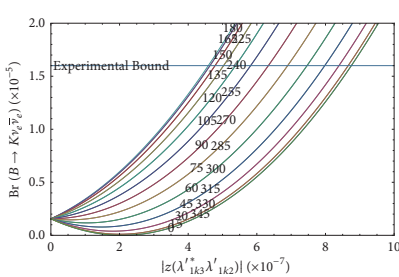

(b)

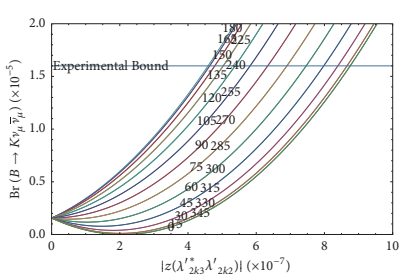

(c)

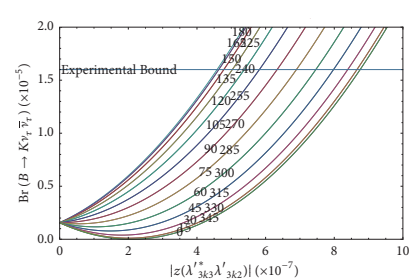

(d)

FiguRE 9: Variations of branching fraction with respect to NP parameter $\left|z\left(\lambda_{i \mathrm{jk}}^{\prime} \lambda_{l \mathrm{mn}}^{\prime}\right)\right|$ at several values of $\theta$ for $B \longrightarrow K \nu_{\alpha} \bar{\nu}_{\alpha} . \alpha$ is (a) unpolarized, (b) e, (c) $\mu$, (d) $\tau$. Experimental bound on the process is $1.6 \times 10^{-5}$. 


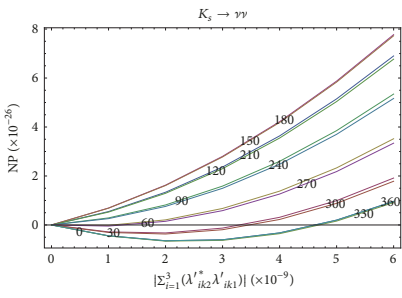

(a)

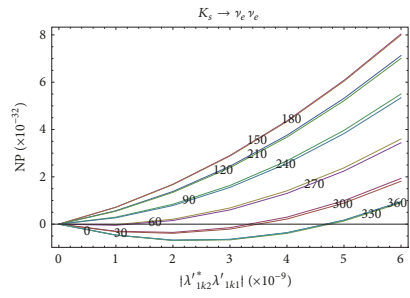

(b)

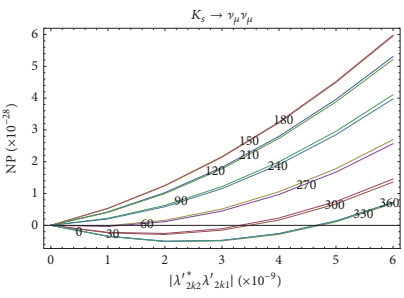

(c)

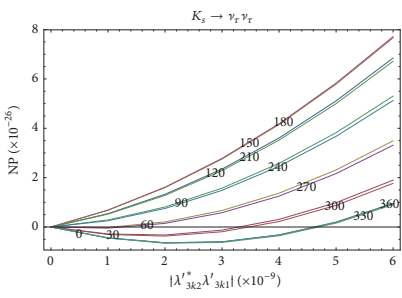

(d)

FIGURE 10: Variations of branching fraction (NP contribution only) with respect to NP parameter $\left|\lambda_{i \mathrm{jk}}^{\prime} \lambda_{l \mathrm{mn}}^{\prime}\right|$ at several values of $\theta$ for $K_{S} \longrightarrow$ $\nu_{\alpha} \bar{\nu}_{\alpha} . \alpha$ is (a) unpolarized, (b) e, (c) $\mu$, (d) $\tau$.

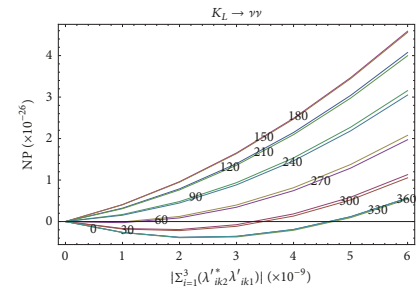

(a)

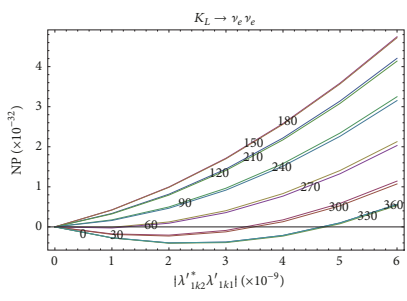

(b)

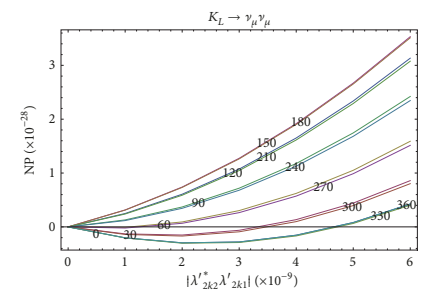

(c)

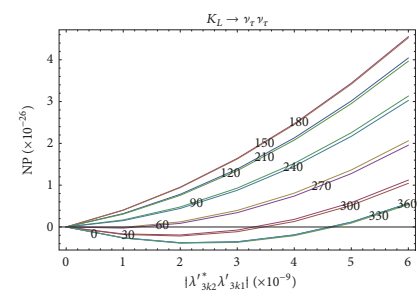

(d)

FIGURE 11: Variations of branching fraction (NP contribution only) with respect to NP parameter $\left|\lambda_{i \mathrm{jk}}^{\prime} \lambda_{l \mathrm{mn}}^{\prime}\right|$ at several values of $\theta$ for $K_{L} \longrightarrow$ $\nu_{\alpha} \bar{\nu}_{\alpha} \cdot \alpha$ is (a) unpolarized, (b) e, (c) $\mu,(\mathrm{d}) \tau$.

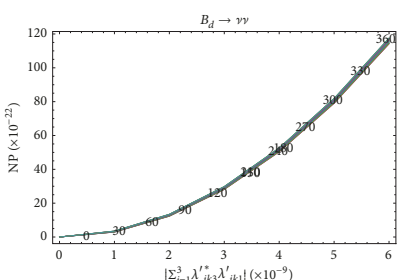

(a)

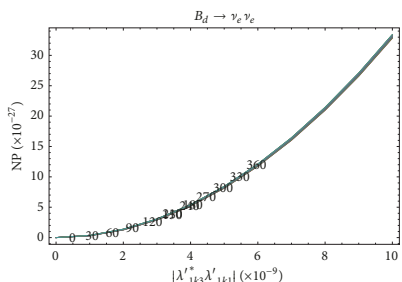

(b)

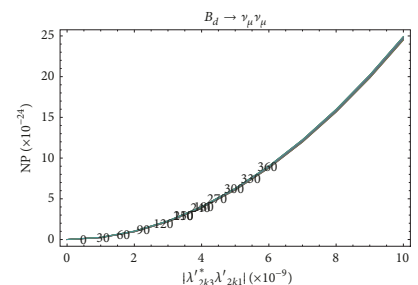

(c)

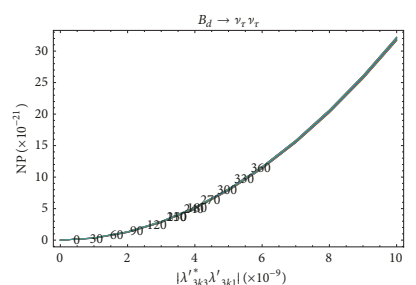

(d)

FIGURE 12: Variations of branching fraction (NP contribution only) with respect to NP parameter $\left|\lambda_{i j \mathrm{k}}^{\prime} \lambda_{l \mathrm{mn}}^{\prime}\right|$ at several values of $\theta$ for $B_{d} \longrightarrow$ $\nu_{\alpha} \bar{\nu}_{\alpha} \cdot \alpha$ is (a) unpolarized, (b) e, (c) $\mu,(\mathrm{d}) \tau$.

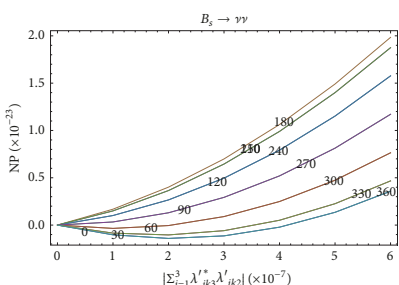

(a)

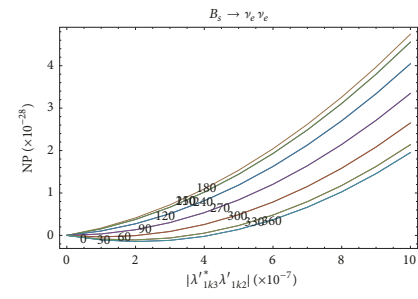

(b)

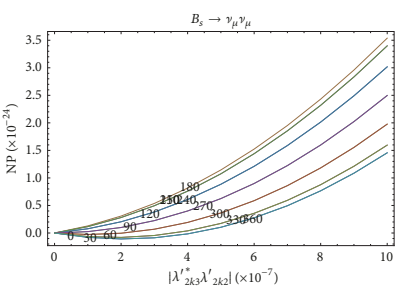

(c)

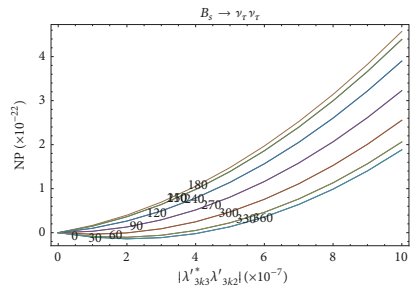

(d)

FIGURE 13: Variations of branching fraction (NP contribution only) with respect to NP parameter $\left|\lambda_{i \mathrm{jk}}^{\prime} \lambda_{l \mathrm{mn}}^{\prime}\right|$ at several values of $\theta$ for $B_{s} \longrightarrow$ $v_{\alpha} \bar{\nu}_{\alpha} . \alpha$ is (a) unpolarized, (b) e, (c) $\mu$, (d) $\tau$. 
TABLE 2: Bounds on NP parameters $\left(\left|z\left(\lambda_{i j \mathrm{k}}^{\prime} \lambda_{l \mathrm{mn}}^{\prime}\right)\right|, \theta\right)$ for $K \longrightarrow \pi v_{\alpha} \bar{\nu}_{\alpha} . \alpha\left(B r_{S M}\right)$ is (a) unpolarized $\left(8.63 \times 10^{-11}\right)$, (b) e $\left(2.89 \times 10^{-11}\right)$,(c) $\mu\left(2.89 \times 10^{-11}\right)$, (d) $\tau\left(2.85 \times 10^{-11}\right)$. Experimental limits are $(1.7 \pm 1.0) \times 10^{-10}$.

\section{(a)}

\begin{tabular}{lc}
\hline & Bounds on NP Parameter (unpolarized) \\
$\theta$ & $\left|z\left(\sum_{i=1}^{3} \lambda_{\mathrm{ik} 2}^{\prime \star} \lambda_{\mathrm{ik} 1}^{\prime}\right)\right| \times 10^{-9}$ \\
\hline 0 & $3.465 \pm 3.209$ \\
30 & $3.032 \pm 2.653$ \\
60 & $4.229 \pm \ldots$. \\
90 & $2.972 \pm \ldots$. \\
120 & $2.233 \pm \ldots$. \\
150 & $1.927 \pm \ldots$. \\
180 & $1.94 \pm \ldots$. \\
210 & $2.278 \pm \ldots$. \\
240 & $3.062 \pm \ldots$. \\
270 & $4.357 \pm \ldots$. \\
300 & $3.078 \pm 2.721$ \\
330 & $3.486 \pm 3.233$ \\
360 & $3.465 \pm 3.209$ \\
\hline
\end{tabular}

(b)

\begin{tabular}{lc}
\hline & Bounds on NP Parameter (unpolarized) \\
$\theta$ & $\left|z\left(\sum_{i=1}^{3} \lambda_{\mathrm{ik} 2}^{\prime \star} \lambda_{\mathrm{ik} 1}^{\prime}\right)\right| \times 10^{-9}$ \\
\hline 0 & $5.575 \pm 1.098$ \\
30 & $4.356 \pm 1.328$ \\
60 & $4.229 \pm \ldots$. \\
90 & $2.972 \pm \ldots$. \\
120 & $2.233 \pm \ldots$. \\
150 & $1.927 \pm \ldots$. \\
180 & $1.94 \pm \ldots$. \\
210 & $2.278 \pm \ldots$. \\
240 & $3.062 \pm \ldots$. \\
270 & $4.357 \pm \ldots$. \\
300 & $4.504 \pm 1.295$ \\
330 & $5.629 \pm 1.09$ \\
360 & $5.575 \pm 1.098$ \\
\hline
\end{tabular}

(c)

\begin{tabular}{lc}
\hline \multicolumn{3}{c}{ Bounds on NP Parameter } \\
$\theta$ & $\left|z\left(\lambda_{1 \mathrm{k} 2}^{\prime \star} \lambda_{1 \mathrm{k} 1}^{\prime}\right)\right| \times 10^{-9}$ \\
\hline 0 & $8.026 \pm 1.87$ \\
30 & $7.083 \pm 1.967$ \\
60 & $5.671 \pm 2.079$ \\
90 & $4.408 \pm 2.072$ \\
120 & $3.618 \pm 1.956$ \\
150 & $3.267 \pm 1.865$ \\
180 & $3.282 \pm 1.87$ \\
\hline
\end{tabular}

(c) Continued.

\begin{tabular}{lc}
\hline \multicolumn{3}{c}{ Bounds on NP Parameter } \\
$\theta$ & $\left|z\left(\lambda_{1 \mathrm{k} 2}^{\prime \star} \lambda_{1 \mathrm{k} 1}^{\prime}\right)\right| \times 10^{-9}$ \\
\hline 210 & $3.666 \pm 1.967$ \\
240 & $4.498 \pm 2.079$ \\
270 & $5.793 \pm 2.072$ \\
300 & $7.189 \pm 1.956$ \\
330 & $8.068 \pm 1.865$ \\
360 & $8.026 \pm 1.87$ \\
\hline
\end{tabular}

(d)

\begin{tabular}{lc}
\hline \multicolumn{3}{c}{ Bounds on NP Parameter } \\
$\theta$ & $\left|z\left(\lambda^{\prime *}{ }_{2 \mathrm{k} 2}^{\prime} \lambda_{2 \mathrm{k} 1}^{\prime}\right)\right| \times 10^{-9}$ \\
\hline 0 & $8.026 \pm 1.87$ \\
30 & $7.083 \pm 1.967$ \\
60 & $5.671 \pm 2.079$ \\
90 & $4.409 \pm 2.072$ \\
120 & $3.618 \pm 1.956$ \\
150 & $3.267 \pm 1.865$ \\
180 & $3.282 \pm 1.87$ \\
210 & $3.666 \pm 1.967$ \\
240 & $4.499 \pm 2.079$ \\
270 & $5.793 \pm 2.072$ \\
300 & $7.189 \pm 1.956$ \\
330 & $8.068 \pm 1.865$ \\
360 & $8.026 \pm 1.87$ \\
\hline
\end{tabular}

(e)

\begin{tabular}{lc}
\hline \multicolumn{3}{c}{ Bounds on NP Parameter } \\
$\theta$ & $\left|\mathrm{z}\left(\lambda_{3 \mathrm{k} 2}^{\prime *} \lambda_{3 \mathrm{k} 1}^{\prime}\right)\right| \times 10^{-9}$ \\
\hline 0 & $8.009 \pm 1.87$ \\
30 & $7.071 \pm 1.966$ \\
60 & $5.671 \pm 2.076$ \\
90 & $4.418 \pm 2.069$ \\
120 & $3.631 \pm 1.954$ \\
150 & $3.282 \pm 1.865$ \\
180 & $3.298 \pm 1.87$ \\
210 & $3.684 \pm 1.966$ \\
240 & $4.515 \pm 2.076$ \\
270 & $5.803 \pm 2.069$ \\
300 & $7.186 \pm 1.954$ \\
330 & $8.054 \pm 1.865$ \\
360 & $8.009 \pm 1.87$ \\
\hline
\end{tabular}

of NP parameter are given in Table 2 . Here the entry “ \pm ........" means that these specific NP parameters cannot be defined 
TABLE 3: Bounds on NP parameters $\left(\left|\lambda_{i \mathrm{j} \times}^{\prime} \lambda_{l \mathrm{mn}}^{\prime}\right|, \theta\right)$ derived from $K \longrightarrow \pi v_{\alpha} \bar{\nu}_{\alpha}$ for $K^{0} \longrightarrow \pi^{0} \nu_{\alpha} \bar{\nu}_{\alpha} \cdot \alpha\left(B r_{S M}\right)$ is (a) unpolarized (2.94. $\left.\times 10^{-11}\right)$ and $9.78 \times 10^{-12}$ for (b) e, (c) $\mu$, (d) $\tau$. Experimental bound on the process is $2.6 \times 10^{-8}$.

(a)

\begin{tabular}{|c|c|c|c|c|}
\hline \multicolumn{2}{|c|}{ Bounds on NP Parameter } & \multicolumn{3}{|c|}{ Branching Fraction $\left(\times 10^{-10}\right)$} \\
\hline$\theta$ & $\left|\sum_{i=1}^{3}\left(\lambda_{\mathrm{ik} 2}^{\prime \star} \lambda_{\mathrm{ik} 1}^{\prime}\right)\right| \times 10^{-9}$ & NP & Interference & Combined \\
\hline 0 & $3.465 \pm 3.209$ & $0 . \pm 0$ & $0 . \pm 0$ & $0.003 \pm 0$ \\
\hline 30 & $3.032 \pm 2.653$ & $2.484 \pm 2.462$ & $-2.446 \pm 2.426$ & $0.041 \pm 0.036$ \\
\hline 60 & $4.229 \pm \ldots$. & $8.212 \pm \ldots$. & $-8.099 \pm \ldots$. & $0.116 \pm \ldots$. \\
\hline 90 & $2.972 \pm \ldots$. & $5.407 \pm \ldots$. & $-5.328 \pm \ldots$. & $0.082 \pm \ldots$. \\
\hline 120 & $2.233 \pm \ldots$. & $2.289 \pm \ldots$. & $-2.25 \pm \ldots$. & $0.042 \pm \ldots$. \\
\hline 150 & $1.927 \pm \ldots$. & $0.568 \pm \ldots$. & $-0.555 \pm \ldots$. & $0.017 \pm \ldots$. \\
\hline 180 & $1.94 \pm \ldots$. & $0 \pm \ldots$ & $0 \pm \ldots$ & $0.003 \pm \ldots$. \\
\hline 210 & $2.278 \pm \ldots$ & $0.794 \pm \ldots$. & $-0.796 \pm \ldots$. & $0.001 \pm \ldots$. \\
\hline 240 & $3.062 \pm \ldots$. & $4.304 \pm \ldots$. & $-4.283 \pm \ldots$. & $0.023 \pm \ldots$. \\
\hline 270 & $4.357 \pm \ldots$. & $11.621 \pm \ldots$. & $-11.541 \pm \ldots$. & $0.082 \pm \ldots$. \\
\hline 300 & $3.078 \pm 2.721$ & $7.75 \pm 7.691$ & $-7.695 \pm 7.634$ & $0.058 \pm 0.057$ \\
\hline 330 & $3.486 \pm 3.233$ & $3.46 \pm 3.45$ & $-3.44 \pm 3.429$ & $0.023 \pm 0.021$ \\
\hline 360 & $3.465 \pm 3.209$ & $0 . \pm 0$ & $0 . \pm 0$ & $0.003 \pm 0$ \\
\hline
\end{tabular}

(b)

\begin{tabular}{lcccc}
\hline \multicolumn{3}{c}{ Bounds on NP Parameter } & \multicolumn{3}{c}{ Branching Fraction $\left(\times 10^{-10}\right)$} \\
$\theta$ & $\left|\lambda_{1 \mathrm{k} 2}^{\prime \star} \lambda_{1 \mathrm{k} 1}^{\prime}\right| \times 10^{-9}$ & NP & Interference & Combined \\
\hline 0 & $8.026 \pm 1.87$ & $0 . \pm 0$. & $0 . \pm 0$. & $0.001 \pm 0$. \\
30 & $7.083 \pm 1.967$ & $2.757 \pm 1.422$ & $-2.719 \pm 1.405$ & $0.039 \pm 0.017$ \\
60 & $5.671 \pm 2.079$ & $5.585 \pm 3.609$ & $-5.515 \pm 3.568$ & $0.071 \pm 0.041$ \\
90 & $4.408 \pm 2.072$ & $4.843 \pm 3.729$ & $-4.782 \pm 3.686$ & $0.062 \pm 0.043$ \\
120 & $3.618 \pm 1.956$ & $2.589 \pm 2.166$ & $-2.554 \pm 2.14$ & $0.036 \pm 0.026$ \\
150 & $3.267 \pm 1.865$ & $0.722 \pm 0.622$ & $-0.71 \pm 0.613$ & $0.013 \pm 0.009$ \\
180 & $3.282 \pm 1.87$ & $0 . \pm 0$. & $0 . \pm 0$. & $0.001 \pm 0$. \\
210 & $3.666 \pm 1.967$ & $0.883 \pm 0.736$ & $-0.879 \pm 0.731$ & $0.005 \pm 0.005$ \\
240 & $4.498 \pm 2.079$ & $3.759 \pm 2.863$ & $-3.732 \pm 2.839$ & $0.028 \pm 0.024$ \\
270 & $5.793 \pm 2.072$ & $7.726 \pm 4.9$ & $-8.429 \pm 4.267$ & $0.062 \pm 0.043$ \\
300 & $7.189 \pm 1.956$ & $8.497 \pm 4.306$ & $-3.475 \pm 1.523$ & $0.068 \pm 0.038$ \\
330 & $8.068 \pm 1.865$ & $3.499 \pm 1.536$ & $0 . \pm 0$. & $0.025 \pm 0.013$ \\
360 & $8.026 \pm 1.87$ & $0 . \pm 0$. & $0.001 \pm 0$. \\
\hline
\end{tabular}

(c)

\begin{tabular}{lcccc}
\hline \multicolumn{3}{c}{ Bounds on NP Parameter } & \multicolumn{3}{c}{ Branching Fraction $\left(\times 10^{-10}\right)$} \\
$\theta$ & $\left|\lambda_{2 \mathrm{k} 2}^{\prime \star} \lambda_{2 \mathrm{k} 1}^{\prime}\right| \times 10^{-9}$ & $\mathrm{NP}$ & Interference & Combined \\
\hline 0 & $8.026 \pm 1.87$ & $0 . \pm 0$. & $0 . \pm 0$. & $0.001 \pm 0$. \\
30 & $7.083 \pm 1.967$ & $2.757 \pm 1.422$ & $-2.719 \pm 1.405$ & $0.039 \pm 0.017$ \\
60 & $5.671 \pm 2.079$ & $5.585 \pm 3.609$ & $-5.515 \pm 3.568$ & $0.071 \pm 0.041$ \\
90 & $4.409 \pm 2.072$ & $4.843 \pm 3.729$ & $-4.782 \pm 3.686$ & $0.062 \pm 0.043$ \\
120 & $3.618 \pm 1.956$ & $2.589 \pm 2.167$ & $-2.554 \pm 2.14$ & $0.036 \pm 0.026$ \\
150 & $3.267 \pm 1.865$ & $0.722 \pm 0.622$ & $-0.71 \pm 0.613$ & $0.013 \pm 0.009$ \\
180 & $3.282 \pm 1.87$ & $0 . \pm 0$. & $0 . \pm 0$. & $0.001 \pm 0$. \\
210 & $3.666 \pm 1.967$ & $0.883 \pm 0.736$ & $-0.88 \pm 0.731$ & $0.005 \pm 0.005$ \\
240 & $4.499 \pm 2.079$ & $3.759 \pm 2.863$ & $-3.732 \pm 2.839$ & $0.028 \pm 0.024$ \\
270 & $5.793 \pm 2.072$ & $7.726 \pm 4.9$ & $-7.665 \pm 4.857$ & $0.062 \pm 0.043$ \\
300 & $7.189 \pm 1.956$ & $8.496 \pm 4.305$ & $-8.429 \pm 4.267$ & $0.068 \pm 0.038$ \\
\hline
\end{tabular}


(c) Continued.

\begin{tabular}{lcccc}
\hline \multicolumn{3}{c}{ Bounds on NP Parameter } & \multicolumn{3}{c}{ Branching Fraction $\left(\times 10^{-10}\right)$} \\
$\theta$ & $\left|\lambda_{2 \mathrm{k} 2}^{\prime *} \lambda_{2 \mathrm{k} 1}^{\prime}\right| \times 10^{-9}$ & NP & Interference & Combined \\
\hline 330 & $8.068 \pm 1.865$ & $3.498 \pm 1.536$ & $-3.475 \pm 1.523$ & $0.025 \pm 0.013$ \\
360 & $8.026 \pm 1.87$ & $0 . \pm 0$. & $0 . \pm 0$. & $0.001 \pm 0$. \\
\hline
\end{tabular}

(d)

\begin{tabular}{lcccc}
\hline \multicolumn{3}{c}{ Bounds on NP Parameter } & \multicolumn{3}{c}{ Branching Fraction $\left(\times 10^{-10}\right)$} \\
$\theta$ & $\left|\lambda_{3 \mathrm{k} 2}^{\prime *} \lambda_{3 \mathrm{k} 1}^{\prime}\right| \times 10^{-9}$ & NP & Interference & Combined \\
\hline 0 & $8.009 \pm 1.87$ & $0 . \pm 0$. & $0 . \pm 0$. & $0.001 \pm 0$. \\
30 & $7.071 \pm 1.966$ & $2.748 \pm 1.418$ & $-2.711 \pm 1.401$ & $0.038 \pm 0.017$ \\
60 & $5.671 \pm 2.076$ & $5.582 \pm 3.603$ & $-5.513 \pm 3.562$ & $0.071 \pm 0.041$ \\
90 & $4.418 \pm 2.069$ & $4.856 \pm 3.73$ & $-4.795 \pm 3.687$ & $0.062 \pm 0.043$ \\
120 & $3.631 \pm 1.954$ & $2.603 \pm 2.173$ & $-2.568 \pm 2.146$ & $0.036 \pm 0.027$ \\
150 & $3.282 \pm 1.865$ & $0.727 \pm 0.625$ & $-0.715 \pm 0.616$ & $0.013 \pm 0.009$ \\
180 & $3.298 \pm 1.87$ & $0 . \pm 0$. & $0 . \pm 0$. & $0.001 \pm 0$. \\
210 & $3.684 \pm 1.966$ & $0.89 \pm 0.739$ & $-0.886 \pm 0.734$ & $0.005 \pm 0.005$ \\
240 & $4.515 \pm 2.076$ & $3.78 \pm 2.869$ & $-3.753 \pm 2.845$ & $0.028 \pm 0.024$ \\
270 & $5.803 \pm 2.069$ & $7.745 \pm 4.899$ & $-7.684 \pm 4.856$ & $0.062 \pm 0.043$ \\
300 & $7.186 \pm 1.954$ & $8.488 \pm 4.299$ & $-8.421 \pm 4.261$ & \\
330 & $8.054 \pm 1.865$ & $3.487 \pm 1.533$ & $-3.464 \pm 1.52$ & $0.068 \pm 0.038$ \\
360 & $8.009 \pm 1.87$ & $0 . \pm 0$. & $0 . \pm 0$. & $0.024 \pm 0.013$ \\
\hline
\end{tabular}

for $-1 \sigma$ as the corresponding $\not_{p}$ MSSM contribution exceeds the experimental limit on branching fraction in $-1 \sigma$ error. Tables 2(a) and 2(b) show the same pattern as observed in Figures 2(a) and 3(a) numerically. Since Yukawa couplings for R-parity violation are identical for the processes $(K \longrightarrow$ $\pi v_{\alpha} \bar{\nu}_{\alpha}, K^{0} \longrightarrow \pi^{0} v_{\alpha} \bar{\nu}_{\alpha}, K_{L, S} \longrightarrow v_{\alpha} \bar{\nu}_{\alpha}$ ), the maximum limits for $K \longrightarrow \pi v_{\alpha} \bar{\nu}_{\alpha}$ are used for calculating NP contribution to branching fraction of other processes. Figure 3 represent the variation of branching fraction with respect to the magnitude of NP parameter at several values of its phase $\theta$.

Contour plots in Figure 4 represent the allowed region for NP parameters $\left(\left|z\left(\lambda_{i k 1}^{\prime} \lambda_{i k 2}^{\prime *}\right)\right|\right.$ and $\left.\theta\right)$ for specific values of branching fraction of the process $K^{0} \longrightarrow \pi^{0} \nu_{\alpha} \bar{\nu}_{a}$. All four plots (comprising unpolarized (a) and polarized (b-d)) show that the maximum magnitude of NP parameter follows a catenary (hanging chain) pattern, with the bottom level smoothening with decreasing error levels. The bounds on the magnitude of NP parameter and possible NP contribution are in Table 3, which shows that $R_{p}$ MSSM dominates over SM contribution by order of magnitude $\sim 10^{2}$, but the overall effect is to give a comparatively less contribution due to destructive interference. The plots in Figure 5 represent the variation of branching fraction (NP contribution only) for the process $K^{0} \longrightarrow \pi^{0} \nu_{\alpha} \bar{\nu}_{a}$ with respect to the magnitude of NP parameter at several values of its phase $\theta$.
Contour graphs in Figure 6 illustrate the allowed region for NP parameters $\left(\left|z\left(\lambda_{i k 1}^{\prime} \lambda_{i k 3}^{\prime *}\right)\right|\right.$ and $\left.\theta\right)$ of the process $B \longrightarrow$ $\pi \nu_{\alpha} \bar{\nu}_{a}$ for specific values of branching fraction. All four plots (comprising unpolarized (a) and (b-d)) show that the maximum magnitude of NP parameter oscillates gently with respect to its phase in general. Similarly, plots in Figure 7 represent the variation of branching fraction with respect to the magnitude of NP parameter $\left(\left|z\left(\lambda_{i k 1}^{\prime} \lambda_{i k 3}^{\prime *}\right)\right|\right)$ at several values of its phase $\theta$. All four plots demonstrate the gentle oscillation behavior as observed in Figure 6 with sharply distinct curves for different values of phases of NP parameter $\theta$. The bounds on the magnitude of NP parameter are given in Table 4.

Contour graphs in Figure 8 depict the allowed region for NP parameters $\left(\left|z\left(\lambda_{i k 3}^{\prime} \lambda_{i k 2}^{\prime *}\right)\right|\right.$ and $\left.\theta\right)$ of the process $B \longrightarrow$ $K \nu_{\alpha} \bar{\nu}_{a}$ for specific values of branching fraction bounded by the experimental limit, while plots in Figure 9 represent the variation of branching fraction of the process $B \longrightarrow K \nu_{\alpha} \bar{\nu}_{a}$ with the magnitude of NP parameters at several values of its phase $\theta$. All four plots (comprising unpolarized (a) and polarized (b-d)) show that the maximum magnitude of NP parameter oscillates with respect to its phase in general. The plot in Figure 8(a) shows a particular pattern below given limiting branching fraction $\left(\leq 4 \times 10^{-6}\right)$. This particular pattern shows that only a narrow range of phase $\theta$ of NP parameter is allowed in that case. The bounds on the magnitude of NP parameter are given in Table 5. 
TABLE 4: Bounds on NP parameters $\left(\left|z\left(\lambda_{i \mathrm{jk}}^{\prime} \lambda_{l \mathrm{mn}}^{\prime}\right)\right|, \theta\right)$ for $B \longrightarrow$ $\pi v_{\alpha} \bar{\nu}_{\alpha} \cdot \alpha\left(B r_{S M}\right)$ is (a) unpolarized $\left(1.73 \times 10^{-7}\right)$ and $5.76 \times 10^{-8}$ for (b) e, (c) $\mu$, (d) $\tau$. Experimental bound on the process is $9.8 \times 10^{-5}$.

(a)

\begin{tabular}{lc}
\hline & Bounds on NP Parameter (unpolarized) \\
$\theta$ & $\left|z\left(\sum_{i=1}^{3} \lambda_{\mathrm{ik} 3}^{\prime \star} \lambda_{\mathrm{ik} 1}^{\prime}\right)\right| \times 10^{-6}$ \\
\hline 0 & 1.0164 \\
30 & 1.0298 \\
60 & 1.0506 \\
90 & 1.0735 \\
120 & 1.0923 \\
150 & 1.1016 \\
180 & 1.0987 \\
210 & 1.0844 \\
240 & 1.0629 \\
270 & 1.0402 \\
300 & 1.0223 \\
330 & 1.0137 \\
360 & 1.0164 \\
\hline
\end{tabular}

(b)

\begin{tabular}{lc}
\hline \multicolumn{3}{c}{ Bounds on NP Parameter } \\
$\theta$ & $\left|z\left(\lambda_{1 \mathrm{k} 3}^{\prime *} \lambda_{1 \mathrm{k} 1}^{\prime}\right)\right| \times 10^{-6}$ \\
\hline 0 & 1.7907 \\
30 & 1.8043 \\
60 & 1.8252 \\
90 & 1.8481 \\
120 & 1.8667 \\
150 & 1.8759 \\
180 & 1.873 \\
210 & 1.8589 \\
240 & 1.8376 \\
270 & 1.8148 \\
300 & 1.7967 \\
330 & 1.7879 \\
360 & 1.7907 \\
\hline
\end{tabular}

(c)

\begin{tabular}{lc}
\hline \multicolumn{3}{c}{ Bounds on NP Parameter } \\
$\theta$ & $\left|z\left(\lambda_{2 \mathrm{k} 3}^{\prime \star} \lambda_{2 \mathrm{k} 1}^{\prime}\right)\right| \times 10^{-6}$ \\
\hline 0 & 1.7907 \\
30 & 1.8043 \\
60 & 1.8252 \\
90 & 1.8481 \\
120 & 1.8667 \\
150 & 1.8759 \\
180 & 1.873 \\
210 & 1.8589 \\
240 & 1.8376 \\
\hline
\end{tabular}

(c) Continued.

\begin{tabular}{lc}
\hline \multicolumn{2}{c}{ Bounds on NP Parameter } \\
$\theta$ & $\left|z\left(\lambda_{2 \mathrm{k} 3}^{\prime \star} \lambda_{2 \mathrm{k} 1}^{\prime}\right)\right| \times 10^{-6}$ \\
\hline 270 & 1.8148 \\
300 & 1.7967 \\
330 & 1.7879 \\
360 & 1.7907 \\
\hline
\end{tabular}

(d)

\begin{tabular}{lc}
\hline \multicolumn{3}{c}{ Bounds on NP Parameter } \\
$\theta$ & $\left|z\left(\lambda_{3 \mathrm{k} 3}^{\prime *} \lambda_{1 \mathrm{k} 1}^{\prime}\right)\right| \times 10^{-6}$ \\
\hline 0 & 1.7907 \\
30 & 1.8043 \\
60 & 1.8252 \\
90 & 1.8481 \\
120 & 1.8667 \\
150 & 1.8759 \\
180 & 1.873 \\
210 & 1.8589 \\
240 & 1.8376 \\
270 & 1.8148 \\
300 & 1.7967 \\
330 & 1.7879 \\
360 & 1.7907 \\
\hline
\end{tabular}

TABLE 5: Bounds on NP parameters $\left(\left|z\left(\lambda_{i \mathrm{jk}}^{\prime} \lambda_{l \mathrm{mn}}^{\prime}\right)\right|, \theta\right)$ for $B \longrightarrow$ $K v_{\alpha} \bar{\nu}_{\alpha} \cdot \alpha\left(B r_{S M}\right)$ is (a) unpolarized $\left(4.69 \times 10^{-6}\right)$ and $\left(1.56 \times 10^{-6}\right)$ for (b) e, (c) $\mu$, (d) $\tau$. Experimental bound on the process is $1.6 \times 10^{-5}$.

(a)

\begin{tabular}{lc}
\hline & Bounds on NP Parameter (unpolarized) \\
$\theta$ & $\left|z\left(\sum_{i=1}^{3} \lambda_{\mathrm{ik3} 3}^{\prime *} \lambda_{\mathrm{ik} 2}^{\prime}\right)\right| \times 10^{-7}$ \\
\hline 0 & 5.9349 \\
30 & 5.512 \\
60 & 4.4436 \\
90 & 3.238 \\
120 & 2.3595 \\
150 & 1.9022 \\
180 & 1.7667 \\
210 & 1.9022 \\
240 & 2.3595 \\
270 & 3.238 \\
300 & 4.4436 \\
330 & 5.512 \\
360 & 5.9349 \\
\hline
\end{tabular}

(b)

Bounds on NP Parameter

\begin{tabular}{lc}
$\theta$ & $\left|z\left(\lambda_{1 \mathrm{k} 3}^{\prime *} \lambda_{1 \mathrm{k} 2}^{\prime}\right)\right| \times 10^{-7}$ \\
\hline 0 & 8.7538 \\
30 & 8.3927 \\
\hline
\end{tabular}


(b) Continued.

\begin{tabular}{lc}
\hline \multicolumn{3}{c}{ Bounds on NP Parameter } \\
$\theta$ & $\left|z\left(\lambda_{1 \mathrm{k} 3}^{\prime *} \lambda_{1 \mathrm{k} 2}^{\prime}\right)\right| \times 10^{-7}$ \\
\hline 60 & 7.4629 \\
90 & 6.3358 \\
120 & 5.3788 \\
150 & 4.7829 \\
180 & 4.5856 \\
210 & 4.7829 \\
240 & 5.3788 \\
270 & 6.3358 \\
300 & 7.4629 \\
330 & 8.3927 \\
360 & 8.7538 \\
\hline
\end{tabular}

(c)

Bounds on NP Parameter

\begin{tabular}{lc}
$\theta$ & $\left|z\left(\lambda^{\prime *}{ }_{2 \mathrm{k} 3}^{\prime} \lambda_{2 \mathrm{k} 2}^{\prime}\right)\right| \times 10^{-7}$ \\
\hline 0 & 8.7538 \\
30 & 8.3927 \\
60 & 7.4629 \\
90 & 6.3358 \\
120 & 5.3788 \\
150 & 4.7829 \\
180 & 4.5856 \\
210 & 4.7829 \\
240 & 5.3788 \\
270 & 6.3358 \\
300 & 7.4629 \\
330 & 8.3927 \\
360 & 8.7538 \\
\hline
\end{tabular}

(d)

Bounds on NP Parameter

\begin{tabular}{lc}
$\theta$ & $\left|z\left(\lambda_{3 \mathrm{k} 3}^{\prime *} \lambda_{3 \mathrm{k} 2}^{\prime}\right)\right| \times 10^{-7}$ \\
\hline 0 & 8.7539 \\
30 & 8.3927 \\
60 & 7.4629 \\
90 & 6.3357 \\
120 & 5.3788 \\
150 & 4.7829 \\
180 & 4.5856 \\
210 & 4.7829 \\
240 & 5.3788 \\
270 & 6.3357 \\
300 & 7.4629 \\
330 & 8.3927 \\
360 & 8.7539 \\
\hline
\end{tabular}

Plots in Figures 10 and 11 of the process $K_{L, S} \longrightarrow v_{\alpha} \bar{\nu}_{\alpha}$ display the variation of branching fraction (NP contribution only) with respect to the magnitude of NP parameter at several values of its phase $\theta$. For pure leptonic decays of strange mesons involving neutrinos $\left(K_{L, S} \longrightarrow \nu_{\alpha} \bar{\nu}_{\alpha}\right)$, there is no experimental data available. Therefore, we use limits derived from $K \longrightarrow \pi v_{\alpha} \bar{\nu}_{\alpha}$ to calculate NP contribution to these processes. The bounds on the magnitude of NP parameter and possible NP contribution are given in Tables 6 and 7 for the decay of $K_{L, S}$, respectively, which shows that $\not R_{p}$ MSSM enhances SM contribution by order of 10 for $K_{L, S}$. The interference term in this case is both constructive and destructive, but the destructive effect is not strong enough to affect the enhancement by SUSY.

Plots in Figures 12 and 13 describe the variation of branching fraction (NP contribution only) of the process $B_{s, d} \longrightarrow \nu_{\alpha} \bar{\nu}_{\alpha}$ with the magnitude of NP parameters, $\left(\left|z\left(\lambda_{i k 1}^{\prime} \lambda_{i k 3}^{\prime *}\right)\right|\right.$ at several values of $\theta$. For pure leptonic decays of beauty involving neutrinos $\left(B_{s, d} \longrightarrow v_{\alpha} \bar{\nu}_{\alpha}\right)$, there is no experimental data available for these processes, and we use limits derived from $B \longrightarrow(\pi, K) \nu_{\alpha} \bar{\nu}_{\alpha}$ to calculate NP contributions to these processes. The bounds on the magnitude of NP parameter and possible NP contribution are given in Tables 8 and 9 , respectively for the decay of $B_{s, d}$, which shows that $R_{p}$ MSSM enhances SM contribution by order of magnitude 10 for $B_{s}$ and $10^{4}$ for $B_{d}$ and also the effect of destructive interference is not strong to affect SUSY enhancement.

\section{Summary and Conclusion}

Summarizing, we have carried out an analysis of semileptonic $\left(M \longrightarrow M^{\prime} v_{a} \bar{\nu}_{\alpha} ; M=K, B ; M^{\prime}=\pi, K ; \alpha=e, \mu, \tau\right)$ and pure leptonic $\left(M \longrightarrow v_{\alpha} \bar{\nu}_{\alpha}\right)$ decays of pseudoscalar mesons within the framework of $R_{p}$ MSSM. The analysis involves a detailed comparison of experimental results with respect to the theoretical prediction of the branching fraction of given processes. The comparison (listed in Tables 2-9) quantifies the effect of contribution from $R_{p}$ MSSM to the branching fraction of processes under discussion. The analysis performed (listed in Tables 3, 6-9) also enables to compare the branching fraction of processes having identical FCNC. The plots in Figures $2-13$ show the variations of branching fraction with respect to NP parameters $\left(\left|z\left(\lambda_{\alpha k \beta}^{\prime} \lambda_{\alpha k \gamma}^{\prime *}\right)\right|\right.$ and $\left.\theta\right)$. In general, SM contribution is dominated by $R_{p}$ MSSM contribution, i.e., by a factor of 10 for the pure leptonic decays of $K_{L, S}$ and by $10^{2}$ and $10^{4}$ in case of $B_{s}$ and $B_{d}$ respectively. The interference term between $R_{p}$ MSSM and SM is both constructive and destructive but except for the semileptonic decays of $K^{0}$, it does not affect the enhancement due to SUSY. This makes $R_{p}$ MSSM a viable model for the comparison of NP contribution in rare decays at labs like Super B factories, KOTO (J-PARC) and NA62 at CERN.

\section{Conflicts of Interest}

The authors declare that there are no conflicts of interest. 
TABLE 6: Bounds on NP parameters $\left(\left|\lambda_{i \mathrm{jk}}^{\prime} \lambda_{l \mathrm{mn}}^{\prime}\right|, \theta\right)$ derived from $K \longrightarrow \pi v_{\alpha} \bar{\nu}_{\alpha}$ for $K_{s} \longrightarrow \nu_{\alpha} \bar{\nu}_{\alpha} \cdot \alpha\left(B r_{S M}\right)$ is (a) unpolarized (7.27 $\left.\times 10^{-27}\right)$, (b) e $\left(7.58 \times 10^{-33}\right),(\mathrm{c}) \mu\left(5.66 \times 10^{-29}\right),(\mathrm{d}) \tau\left(7.27 \times 10^{-27}\right)$.

(a)

\begin{tabular}{|c|c|c|c|c|}
\hline \multicolumn{2}{|c|}{ Bounds on NP Parameter } & \multicolumn{3}{|c|}{ Branching Fraction $\left(\times 10^{-26}\right)$} \\
\hline$\theta$ & $\left|\sum_{i=1}^{3}\left(\lambda_{\mathrm{ik} 2}^{\prime *} \lambda_{\mathrm{ik} 1}^{\prime}\right)\right| \times 10^{-9}$ & NP & Interference & Combined \\
\hline 0 & $3.465 \pm 3.209$ & $2.69 \pm 2.682$ & $-1.952 \pm 1.808$ & $1.454 \pm 0.873$ \\
\hline 30 & $3.032 \pm 2.653$ & $1.958 \pm 1.94$ & $-0.764 \pm 0.669$ & $1.91 \pm 1.272$ \\
\hline 60 & $4.229 \pm \ldots$ & $2.157 \pm \ldots$ & $2.055 \pm \ldots$. & $4.928 \pm \ldots$. \\
\hline 90 & $2.972 \pm \ldots$. & $1.065 \pm \ldots$. & $1.195 \pm \ldots$. & $2.976 \pm \ldots$. \\
\hline 120 & $2.233 \pm \ldots$ & $0.601 \pm \ldots$. & $-0.808 \pm \ldots$ & $0.509 \pm \ldots$. \\
\hline 150 & $1.927 \pm \ldots$ & $0.448 \pm \ldots$ & $-0.989 \pm \ldots$. & $0.175 \pm \ldots$ \\
\hline 180 & $1.94 \pm \ldots$. & $0.454 \pm \ldots$. & $0.395 \pm \ldots$ & $1.565 \pm \ldots$. \\
\hline 210 & $2.278 \pm \ldots$. & $0.626 \pm \ldots$ & $1.312 \pm \ldots$ & $2.654 \pm \ldots$. \\
\hline 240 & $3.062 \pm \ldots$. & $1.131 \pm \ldots$ & $-0.079 \pm \ldots$ & $1.768 \pm \ldots$. \\
\hline 270 & $4.357 \pm \ldots$. & $2.289 \pm \ldots$. & $-2.545 \pm \ldots$ & $0.46 \pm \ldots$. \\
\hline 300 & $3.078 \pm 2.721$ & $2.035 \pm 2.021$ & $-0.476 \pm 0.421$ & $2.276 \pm 1.6$ \\
\hline 330 & $3.486 \pm 3.233$ & $2.726 \pm 2.718$ & $1.87 \pm 1.734$ & $5.313 \pm 4.453$ \\
\hline 360 & $3.465 \pm 3.209$ & $2.69 \pm 2.682$ & $1.109 \pm 1.027$ & $4.515 \pm 3.709$ \\
\hline
\end{tabular}

(b)

\begin{tabular}{lcccc}
\hline \multicolumn{3}{c}{ Bounds on NP Parameter } & \multicolumn{3}{c}{ Branching Fraction $\left(\times 10^{-32}\right)$} \\
$\theta$ & $\left|\lambda_{1 \mathrm{k} 2}^{\prime *} \lambda_{1 \mathrm{k} 1}^{\prime}\right| \times 10^{-9}$ & $\mathrm{NP}$ & Interference & Combined \\
\hline 0 & $8.026 \pm 1.87$ & $8.435 \pm 3.725$ & $-4.716 \pm 1.097$ & $-1.846 \pm 0.513$ \\
30 & $7.083 \pm 1.967$ & $6.71 \pm 3.46$ & $2.876 \pm 1.052$ & $5.618 \pm 2.947$ \\
60 & $5.671 \pm 2.079$ & $4.53 \pm 2.93$ & $1.834 \pm 0.862$ & $5.16 \pm 3.982$ \\
90 & $4.408 \pm 2.072$ & $2.95 \pm 2.27$ & $-1.368 \pm 0.742$ & $1.538 \pm 3.132$ \\
120 & $3.618 \pm 1.956$ & $2.1 \pm 1.76$ & $-1.741 \pm 0.997$ & $0.768 \pm 0.518$ \\
150 & $3.267 \pm 1.865$ & $1.755 \pm 1.515$ & $0.703 \pm 0.403$ & $3.227 \pm 1.923$ \\
180 & $3.282 \pm 1.87$ & $1.77 \pm 1.52$ & $2.198 \pm 1.18$ & $5.102 \pm 2.97$ \\
210 & $3.666 \pm 1.967$ & $2.15 \pm 1.79$ & $-0.131 \pm 0.058$ & $3.673 \pm 2.262$ \\
240 & $4.498 \pm 2.079$ & $3.05 \pm 2.32$ & $-3.524 \pm 1.259$ & $1.93 \pm 1.72$ \\
270 & $5.793 \pm 2.072$ & $4.7 \pm 2.98$ & $-1.145 \pm 0.314$ & $6.504 \pm 3.181$ \\
300 & $7.189 \pm 1.956$ & $6.895 \pm 3.495$ & $4.513 \pm 1.045$ & $13.782 \pm 4.78$ \\
330 & $8.068 \pm 1.865$ & $8.515 \pm 3.735$ & $2.66 \pm 0.622$ & $11.848 \pm 4.347$ \\
360 & $8.026 \pm 1.87$ & $8.435 \pm 3.725$ & & 1.018 \\
\hline
\end{tabular}

(c)

\begin{tabular}{lcccc}
\hline \multicolumn{3}{c}{ Bounds on NP Parameter } & \multicolumn{3}{c}{ Branching Fraction $\left(\times 10^{-28}\right)$} \\
$\theta$ & $\left|\lambda_{2 \mathrm{k} 2}^{\prime *} \lambda_{2 \mathrm{k} 1}^{\prime}\right| \times 10^{-9}$ & $\mathrm{NP}$ & Interference & Combined \\
\hline 0 & $8.026 \pm 1.87$ & $6.29 \pm 2.78$ & $-3.497 \pm 0.815$ & $3.35 \pm 1.964$ \\
30 & $7.083 \pm 1.967$ & $5.004 \pm 2.58$ & $-1.374 \pm 0.381$ & $4.187 \pm 2.199$ \\
60 & $5.671 \pm 2.079$ & $3.379 \pm 2.184$ & $2.132 \pm 0.782$ & $6.068 \pm 2.965$ \\
90 & $4.409 \pm 2.072$ & $2.198 \pm 1.692$ & $1.366 \pm 0.642$ & $4.12 \pm 2.334$ \\
120 & $3.618 \pm 1.956$ & $1.566 \pm 1.311$ & $-1.014 \pm 0.548$ & $0.11 \pm 0.762$ \\
150 & $3.267 \pm 1.865$ & $1.31 \pm 1.128$ & $-1.295 \pm 0.739$ & $2.396 \pm 1.432$ \\
180 & $3.282 \pm 1.87$ & $1.322 \pm 1.136$ & $0.518 \pm 0.296$ & $3.792 \pm 2.211$ \\
210 & $3.666 \pm 1.967$ & $1.603 \pm 1.336$ & $1.632 \pm 0.875$ & $2.738 \pm 1.689$ \\
240 & $4.499 \pm 2.079$ & $2.274 \pm 1.732$ & $-0.093 \pm 0.043$ & \\
270 & $5.793 \pm 2.072$ & $3.506 \pm 2.224$ & $-2.616 \pm 0.936$ & \\
300 & $7.189 \pm 1.956$ & $5.141 \pm 2.605$ & $-0.853 \pm 0.232$ & \\
\hline
\end{tabular}


(c) Continued.

\begin{tabular}{lcccr}
\hline \multicolumn{3}{c}{ Bounds on NP Parameter } & \multicolumn{3}{c}{ Branching Fraction $\left(\times 10^{-28}\right)$} \\
$\theta$ & $\left|\lambda_{2 \mathrm{k} 2}^{\prime *} \lambda_{2 \mathrm{k} 1}^{\prime}\right| \times 10^{-9}$ & NP & Interference & Combined \\
\hline 330 & $8.068 \pm 1.865$ & $6.35 \pm 2.787$ & $3.346 \pm 0.773$ & $10.254 \pm 3.561$ \\
360 & $8.026 \pm 1.87$ & $6.29 \pm 2.78$ & $1.979 \pm 0.461$ & $8.826 \pm 3.241$ \\
\hline
\end{tabular}

(d)

\begin{tabular}{lcccc}
\hline \multicolumn{3}{c}{ Bounds on NP Parameter } & \multicolumn{3}{c}{ Branching Fraction $\left(\times 10^{-26}\right)$} \\
$\theta$ & $\left|\lambda_{3 \mathrm{k} 2}^{\prime *} \lambda_{3 \mathrm{k} 1}^{\prime}\right| \times 10^{-9}$ & NP & Interference & Combined \\
\hline 0 & $8.009 \pm 1.87$ & $8.097 \pm 3.584$ & $-4.48 \pm 1.046$ & $4.328 \pm 2.539$ \\
30 & $7.071 \pm 1.966$ & $6.448 \pm 3.328$ & $-1.768 \pm 0.491$ & $5.39 \pm 2.836$ \\
60 & $5.671 \pm 2.076$ & $4.365 \pm 2.818$ & $2.734 \pm 1$. & $7.81 \pm 3.818$ \\
90 & $4.418 \pm 2.069$ & $2.849 \pm 2.188$ & $1.761 \pm 0.825$ & $5.32 \pm 3.012$ \\
120 & $3.631 \pm 1.954$ & $2.035 \pm 1.698$ & $-1.304 \pm 0.701$ & $1.442 \pm 0.997$ \\
150 & $3.282 \pm 1.865$ & $1.706 \pm 1.466$ & $-1.672 \pm 0.951$ & $0.744 \pm 0.515$ \\
180 & $3.298 \pm 1.87$ & $1.72 \pm 1.477$ & $0.666 \pm 0.378$ & $3.097 \pm 1.854$ \\
210 & $3.684 \pm 1.966$ & $2.087 \pm 1.734$ & $2.106 \pm 1.124$ & $4.904 \pm 2.858$ \\
240 & $4.515 \pm 2.076$ & $2.955 \pm 2.244$ & $-0.115 \pm 0.053$ & $3.552 \pm 2.19$ \\
270 & $5.803 \pm 2.069$ & $4.542 \pm 2.874$ & $-3.364 \pm 1.2$ & $1.889 \pm 1.674$ \\
300 & $7.186 \pm 1.954$ & $6.638 \pm 3.362$ & $-1.102 \pm 0.299$ & $6.247 \pm 3.063$ \\
330 & $8.054 \pm 1.865$ & $8.181 \pm 3.596$ & $4.288 \pm 0.992$ & $13.18 \pm 4.589$ \\
360 & $8.009 \pm 1.87$ & $8.097 \pm 3.584$ & $2.543 \pm 0.594$ & $11.35 \pm 4.178$ \\
\hline
\end{tabular}

TABLE 7: Bounds on NP parameters $\left(\left|\lambda_{i \mathrm{jk}}^{\prime} \lambda_{l \mathrm{mn}}^{\prime}\right|, \theta\right)$ derived from $K \longrightarrow \pi v_{\alpha} \bar{\nu}_{\alpha}$ for $K_{L} \longrightarrow v_{\alpha} \bar{\nu}_{\alpha} . \alpha\left(B r_{S M}\right)$ is (a) unpolarized (4.29 $\left.\times 10^{-27}\right),(\mathrm{b})$ e $\left(4.48 \times 10^{-33}\right),(\mathrm{c}) \mu\left(3.34 \times 10^{-29}\right),(\mathrm{d}) \tau\left(4.26 \times 10^{-27}\right)$.

(a)

\begin{tabular}{|c|c|c|c|c|}
\hline \multicolumn{2}{|c|}{ Bounds on NP Parameter } & \multicolumn{3}{|c|}{ Branching Fraction $\left(\times 10^{-26}\right)$} \\
\hline$\theta$ & $\left|\sum_{i=1}^{3}\left(\lambda_{\mathrm{ik} 2}^{\prime \star} \lambda_{\mathrm{ik} 1}^{\prime}\right)\right| \times 10^{-9}$ & NP & Interference & Combined \\
\hline 0 & $3.465 \pm 3.209$ & $1.588 \pm 1.583$ & $-1.153 \pm 1.067$ & $0.858 \pm 0.516$ \\
\hline 30 & $3.032 \pm 2.653$ & $1.156 \pm 1.146$ & $-0.451 \pm 0.395$ & $1.128 \pm 0.75$ \\
\hline 60 & $4.229 \pm \ldots$. & $1.274 \pm \ldots$ & $1.213 \pm \ldots$ & $2.91 \pm \ldots$ \\
\hline 90 & $2.972 \pm \ldots$ & $0.629 \pm \ldots$. & $0.705 \pm \ldots$ & $1.757 \pm \ldots$. \\
\hline 120 & $2.233 \pm \ldots$. & $0.355 \pm \ldots$ & $-0.477 \pm \ldots$ & $0.301 \pm \ldots$. \\
\hline 150 & $1.927 \pm \ldots$ & $0.264 \pm \ldots$ & $-0.584 \pm \ldots$. & $0.103 \pm \ldots$ \\
\hline 180 & $1.94 \pm \ldots$. & $0.268 \pm \ldots$ & $0.233 \pm \ldots$. & $0.924 \pm \ldots$. \\
\hline 210 & $2.278 \pm \ldots$ & $0.369 \pm \ldots$ & $0.775 \pm \ldots$ & $1.567 \pm \ldots$. \\
\hline 240 & $3.062 \pm \ldots$. & $0.667 \pm \ldots$. & $-0.046 \pm \ldots$. & $1.044 \pm \ldots$. \\
\hline 270 & $4.357 \pm \ldots$. & $1.352 \pm \ldots$. & $-1.503 \pm \ldots$ & $0.272 \pm \ldots$. \\
\hline 300 & $3.078 \pm 2.721$ & $1.202 \pm 1.193$ & $-0.281 \pm 0.248$ & $1.344 \pm 0.945$ \\
\hline 330 & $3.486 \pm 3.233$ & $1.61 \pm 1.605$ & $1.104 \pm 1.024$ & $3.136 \pm 2.63$ \\
\hline 360 & $3.465 \pm 3.209$ & $1.588 \pm 1.583$ & $0.655 \pm 0.606$ & $2.666 \pm 2.19$ \\
\hline
\end{tabular}

(b)

\begin{tabular}{lcccc}
\hline \multicolumn{3}{c}{ Bounds on NP Parameter } & \multicolumn{2}{c}{ Branching Fraction $\left(\times 10^{-32}\right)$} \\
$\theta$ & $\left|\lambda_{1 \mathrm{k} 2}^{\prime{ }^{\star}} \lambda_{1 \mathrm{k} 1}^{\prime}\right| \times 10^{-9}$ & $\mathrm{NP}$ & Interference & Combined \\
\hline 0 & $8.026 \pm 1.87$ & $4.98 \pm 2.2$ & $-2.784 \pm 0.648$ & $2.641 \pm 1.552$ \\
30 & $7.083 \pm 1.967$ & $3.96 \pm 2.04$ & $-1.088 \pm 0.3$ & $3.317 \pm 1.74$ \\
60 & $5.671 \pm 2.079$ & $2.675 \pm 1.725$ & $1.698 \pm 0.626$ & $4.818 \pm 2.351$ \\
\hline
\end{tabular}


(b) Continued.

\begin{tabular}{lcccc}
\hline \multicolumn{3}{c}{ Bounds on NP Parameter } & \multicolumn{3}{c}{ Branching Fraction $\left(\times 10^{-32}\right)$} \\
$\theta$ & $\left|\lambda_{1 \mathrm{k} 2}^{\prime \star} \lambda_{1 \mathrm{k} 1}^{\prime}\right| \times 10^{-9}$ & $\mathrm{NP}$ & \multicolumn{1}{c}{ Interference } & Combined \\
\hline 90 & $4.408 \pm 2.072$ & $1.74 \pm 1.34$ & $1.085 \pm 0.51$ & $3.27 \pm 1.85$ \\
120 & $3.618 \pm 1.956$ & $1.24 \pm 1.04$ & $-0.808 \pm 0.439$ & $0.877 \pm 0.601$ \\
150 & $3.267 \pm 1.865$ & $1.035 \pm 0.895$ & $-1.026 \pm 0.589$ & $0.454 \pm 0.306$ \\
180 & $3.282 \pm 1.87$ & $1.05 \pm 0.9$ & $0.41 \pm 0.235$ & $1.906 \pm 1.136$ \\
210 & $3.666 \pm 1.967$ & $1.27 \pm 1.06$ & $1.297 \pm 0.694$ & $3.012 \pm 1.754$ \\
240 & $4.498 \pm 2.079$ & $1.8 \pm 1.37$ & $-0.076 \pm 0.034$ & $2.169 \pm 1.336$ \\
270 & $5.793 \pm 2.072$ & $2.775 \pm 1.765$ & $-2.081 \pm 0.749$ & \\
300 & $7.189 \pm 1.956$ & $4.07 \pm 2.06$ & $-0.674 \pm 0.181$ & $3.139 \pm 1.016$ \\
330 & $8.068 \pm 1.865$ & $5.025 \pm 2.205$ & $2.668 \pm 0.617$ & \\
360 & $8.026 \pm 1.87$ & $4.98 \pm 2.2$ & $1.57 \pm 0.366$ & \\
\hline
\end{tabular}

(c)

\begin{tabular}{lcccc}
\hline \multicolumn{3}{c}{ Bounds on NP Parameter } & \multicolumn{3}{c}{ Branching Fraction $\left(\times 10^{-28}\right)$} & Interference & Combined \\
$\theta$ & $\left|\lambda_{2 \mathrm{k} 2}^{\prime *} \lambda_{2 \mathrm{k} 1}^{\prime}\right| \times 10^{-9}$ & $\mathrm{NP}$ & $-2.064 \pm 0.481$ & $1.978 \pm 1.16$ \\
\hline 0 & $8.026 \pm 1.87$ & $3.713 \pm 1.641$ & $-0.811 \pm 0.225$ & $2.472 \pm 1.298$ \\
30 & $7.083 \pm 1.967$ & $2.954 \pm 1.523$ & $1.259 \pm 0.461$ & $3.582 \pm 1.751$ \\
60 & $5.671 \pm 2.079$ & $1.995 \pm 1.289$ & $0.806 \pm 0.378$ & $2.433 \pm 1.378$ \\
90 & $4.409 \pm 2.072$ & $1.298 \pm 1$. & $-0.599 \pm 0.324$ & $0.655 \pm 0.45$ \\
120 & $3.618 \pm 1.956$ & $0.925 \pm 0.774$ & $-0.764 \pm 0.436$ & $0.338 \pm 0.23$ \\
150 & $3.267 \pm 1.865$ & $0.774 \pm 0.666$ & $0.306 \pm 0.175$ & $1.414 \pm 0.846$ \\
180 & $3.282 \pm 1.87$ & $0.78 \pm 0.671$ & $0.963 \pm 0.517$ & $2.238 \pm 1.305$ \\
210 & $3.666 \pm 1.967$ & $0.946 \pm 0.789$ & $-0.055 \pm 0.025$ & $1.616 \pm 0.997$ \\
240 & $4.499 \pm 2.079$ & $1.342 \pm 1.023$ & $-1.544 \pm 0.552$ & $0.854 \pm 0.76$ \\
270 & $5.793 \pm 2.072$ & $2.07 \pm 1.313$ & $-0.503 \pm 0.136$ & $2.86 \pm 1.402$ \\
300 & $7.189 \pm 1.956$ & $3.035 \pm 1.538$ & $1.976 \pm 0.457$ & $6.054 \pm 2.102$ \\
330 & $8.068 \pm 1.865$ & $3.75 \pm 1.646$ & $1.169 \pm 0.273$ & $5.211 \pm 1.914$ \\
360 & $8.026 \pm 1.87$ & $3.713 \pm 1.641$ & & \\
\hline
\end{tabular}

(d)

\begin{tabular}{lcccc}
\hline \multicolumn{3}{c}{ Bounds on NP Parameter } & & Branching Fraction $\left(\times 10^{-26}\right)$ \\
$\theta$ & $\left|\lambda_{3 \mathrm{k} 2}^{\prime \star} \lambda_{3 \mathrm{k} 1}^{\prime}\right| \times 10^{-9}$ & $\mathrm{NP}$ & Interference & Combined \\
\hline 0 & $8.009 \pm 1.87$ & $4.78 \pm 2.116$ & $-2.646 \pm 0.617$ & $2.555 \pm 1.499$ \\
30 & $7.071 \pm 1.966$ & $3.806 \pm 1.964$ & $-1.044 \pm 0.29$ & $3.182 \pm 1.674$ \\
60 & $5.671 \pm 2.076$ & $2.577 \pm 1.663$ & $1.614 \pm 0.591$ & $4.611 \pm 2.254$ \\
90 & $4.418 \pm 2.069$ & $1.682 \pm 1.291$ & $1.04 \pm 0.487$ & $3.141 \pm 1.778$ \\
120 & $3.631 \pm 1.954$ & $1.202 \pm 1.003$ & $-0.77 \pm 0.414$ & $0.851 \pm 0.588$ \\
150 & $3.282 \pm 1.865$ & $1.007 \pm 0.865$ & $-0.988 \pm 0.561$ & $0.439 \pm 0.304$ \\
180 & $3.298 \pm 1.87$ & $1.016 \pm 0.871$ & $0.393 \pm 0.223$ & $1.828 \pm 1.094$ \\
210 & $3.684 \pm 1.966$ & $1.233 \pm 1.024$ & $1.243 \pm 0.664$ & $2.896 \pm 1.688$ \\
240 & $4.515 \pm 2.076$ & $1.746 \pm 1.325$ & $-0.069 \pm 0.031$ & $2.096 \pm 1.294$ \\
270 & $5.803 \pm 2.069$ & $2.682 \pm 1.696$ & $-1.986 \pm 0.708$ & \\
300 & $7.186 \pm 1.954$ & $3.919 \pm 1.985$ & $-0.651 \pm 0.177$ & \\
330 & $8.054 \pm 1.865$ & $4.83 \pm 2.123$ & $2.531 \pm 0.586$ & \\
360 & $8.009 \pm 1.87$ & $4.78 \pm 2.116$ & $1.501 \pm 0.351$ & \\
\hline
\end{tabular}




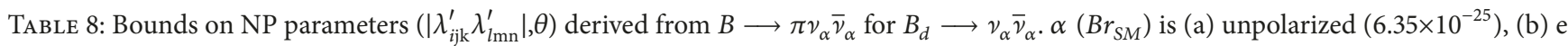
$\left(6.53 \times 10^{-31}\right),(\mathrm{c}) \mu\left(4.87 \times 10^{-27}\right),(\mathrm{d}) \tau\left(6.3 \times 10^{-25}\right)$

(a)

\begin{tabular}{lcccc}
\hline \multicolumn{3}{c}{ Bounds on NP Parameter } & \multicolumn{2}{c}{ Branching Fraction $\left(\times 10^{-22}\right)$} \\
$\theta$ & $\left|\sum_{i=1}^{3} \lambda_{i \mathrm{ik} 3}^{\prime *} \lambda_{\mathrm{ik} 1}^{\prime}\right| \times 10^{-6}$ & NP & Interference & 3.269 \\
\hline 0 & 1.0164 & 3.325 & 0.151 & 3.57 \\
30 & 1.0298 & 3.413 & -0.23 & 3.328 \\
60 & 1.0506 & 3.552 & -0.23 & 3.485 \\
90 & 1.0735 & 3.709 & 0.168 & 4.014 \\
120 & 1.0923 & 3.84 & 0.289 & 3.2 \\
150 & 1.1016 & 3.905 & -0.08 & 3.811 \\
180 & 1.0987 & 3.885 & -0.308 & 3.482 \\
210 & 1.0844 & 3.784 & -0.016 \\
240 & 1.0629 & 3.636 & 0.291 \\
270 & 1.0402 & 3.482 & 0.104 \\
300 & 1.0223 & 3.363 & -0.252 \\
330 & 1.0137 & 3.307 & -0.181 \\
360 & 1.0164 & 3.325 & 3.626 \\
\hline
\end{tabular}

(b)

\begin{tabular}{|c|c|c|c|c|}
\hline \multicolumn{2}{|c|}{ Bounds on NP Parameter } & \multicolumn{3}{|c|}{ Branching Fraction $\left(\times 10^{-27}\right)$} \\
\hline$\theta$ & $\left|\lambda_{1 \mathrm{k} 3}^{\prime \star} \lambda_{1 \mathrm{k} 1}^{\prime}\right| \times 10^{-6}$ & NP & Interference & Combined \\
\hline 0 & 1.7907 & 1.06 & 0.051 & 1.112 \\
\hline 30 & 1.8043 & 1.08 & 0.025 & 1.106 \\
\hline 60 & 1.8252 & 1.1 & -0.038 & 1.063 \\
\hline 90 & 1.8481 & 1.13 & -0.04 & 1.091 \\
\hline 120 & 1.8667 & 1.15 & 0.034 & 1.185 \\
\hline 150 & 1.8759 & 1.17 & 0.046 & 1.217 \\
\hline 180 & 1.873 & 1.16 & -0.012 & 1.149 \\
\hline 210 & 1.8589 & 1.14 & -0.05 & 1.091 \\
\hline 240 & 1.8376 & 1.12 & -0.005 & 1.116 \\
\hline 270 & 1.8148 & 1.09 & 0.053 & 1.144 \\
\hline 300 & 1.7967 & 1.07 & 0.018 & 1.089 \\
\hline 330 & 1.7879 & 1.06 & -0.047 & 1.014 \\
\hline \multirow[t]{3}{*}{360} & 1.7907 & 1.06 & -0.031 & 1.03 \\
\hline & & \multicolumn{3}{|l|}{ (c) } \\
\hline & Bounds on NP Parameter & \multicolumn{3}{|c|}{ Branching Fraction $\left(\times 10^{-23}\right)$} \\
\hline$\theta$ & $\left|\lambda_{2 \mathrm{k} 3}^{\prime *} \lambda_{2 \mathrm{k} 1}^{\prime}\right| \times 10^{-6}$ & NP & Interference & Combined \\
\hline 0 & 1.7907 & 0.792 & 0.037 & 0.829 \\
\hline 30 & 1.8043 & 0.804 & 0.021 & 0.825 \\
\hline 60 & 1.8252 & 0.823 & -0.03 & 0.793 \\
\hline 90 & 1.8481 & 0.844 & -0.03 & 0.814 \\
\hline 120 & 1.8667 & 0.861 & 0.022 & 0.883 \\
\hline 150 & 1.8759 & 0.87 & 0.038 & 0.908 \\
\hline 180 & 1.873 & 0.867 & -0.01 & 0.857 \\
\hline 210 & 1.8589 & 0.854 & -0.04 & 0.814 \\
\hline 240 & 1.8376 & 0.834 & -0.001 & 0.833 \\
\hline 270 & 1.8148 & 0.814 & 0.039 & 0.853 \\
\hline 300 & 1.7967 & 0.798 & 0.014 & 0.812 \\
\hline
\end{tabular}


(c) Continued.

\begin{tabular}{|c|c|c|c|c|}
\hline \multicolumn{2}{|c|}{ Bounds on NP Parameter } & \multicolumn{3}{|c|}{ Branching Fraction $\left(\times 10^{-23}\right)$} \\
\hline$\theta$ & $\left|\lambda_{2 \mathrm{k} 3}^{\prime *} \lambda_{2 \mathrm{k} 1}^{\prime}\right| \times 10^{-6}$ & NP & Interference & Combined \\
\hline 330 & 1.7879 & 0.79 & -0.034 & 0.756 \\
\hline 360 & 1.7907 & 0.792 & -0.024 & 0.768 \\
\hline \multicolumn{5}{|c|}{ (d) } \\
\hline \multicolumn{2}{|c|}{ Bounds on NP Parameter } & \multicolumn{3}{|c|}{ Branching Fraction $\left(\times 10^{-21}\right)$} \\
\hline$\theta$ & $\left|\lambda_{3 \mathrm{k} 3}^{\prime \star} \lambda_{3 \mathrm{k} 1}^{\prime}\right| \times 10^{-6}$ & NP & Interference & Combined \\
\hline 0 & 1.7907 & 1.024 & 0.047 & 1.072 \\
\hline 30 & 1.8043 & 1.04 & 0.026 & 1.067 \\
\hline 60 & 1.8252 & 1.064 & -0.04 & 1.025 \\
\hline 90 & 1.8481 & 1.091 & -0.04 & 1.052 \\
\hline 120 & 1.8667 & 1.113 & 0.028 & 1.142 \\
\hline 150 & 1.8759 & 1.124 & 0.048 & 1.173 \\
\hline 180 & 1.873 & 1.12 & -0.014 & 1.107 \\
\hline 210 & 1.8589 & 1.103 & -0.052 & 1.052 \\
\hline 240 & 1.8376 & 1.078 & -0.003 & 1.076 \\
\hline 270 & 1.8148 & 1.052 & 0.05 & 1.103 \\
\hline 300 & 1.7967 & 1.031 & 0.018 & 1.05 \\
\hline 330 & 1.7879 & 1.021 & -0.045 & 0.977 \\
\hline 360 & 1.7907 & 1.024 & -0.032 & 0.993 \\
\hline
\end{tabular}

TABLE 9: Bounds on NP parameters $\left(\left|\lambda_{i \mathrm{jk}}^{\prime} \lambda_{l \mathrm{mn}}^{\prime}\right|, \theta\right)$ derived from $B \longrightarrow K \nu_{\alpha} \bar{\nu}_{\alpha}$ for $B_{s} \longrightarrow v_{\alpha} \bar{\nu}_{\alpha} \cdot \alpha\left(B r_{S M}\right)$ is (a) unpolarized (1.41 $\left.\times 10^{-23}\right)$, (b) e $\left(1.45 \times 10^{-29}\right)$, (c) $\mu\left(1.08 \times 10^{-25}\right)$, (d) $\tau\left(1.4 \times 10^{-23}\right)$.

(a)

\begin{tabular}{|c|c|c|c|c|}
\hline \multicolumn{2}{|c|}{ Bounds on NP Parameter } & \multicolumn{3}{|c|}{ Branching Fraction $\left(\times 10^{-22}\right)$} \\
\hline$\theta$ & $\left|\sum_{i=1}^{3}\left(\lambda_{\mathrm{ik} 3}^{\prime \star} \lambda_{\mathrm{ik} 2}^{\prime}\right)\right| \times 10^{-7}$ & NP & Interference & Combined \\
\hline 0 & 5.9349 & 1.145 & -0.804 & 0.482 \\
\hline 30 & 5.512 & 0.988 & -0.115 & 1.014 \\
\hline 60 & 4.4436 & 0.642 & 0.574 & 1.357 \\
\hline 90 & 3.238 & 0.341 & 0.197 & 0.679 \\
\hline 120 & 2.3595 & 0.181 & -0.26 & 0.062 \\
\hline 150 & 1.9022 & 0.118 & -0.18 & 0.079 \\
\hline 180 & 1.7667 & 0.101 & 0.144 & 0.386 \\
\hline 210 & 1.9022 & 0.118 & 0.228 & 0.487 \\
\hline 240 & 2.3595 & 0.181 & -0.104 & 0.218 \\
\hline 270 & 3.238 & 0.341 & -0.432 & 0.05 \\
\hline 300 & 4.4436 & 0.642 & 0.014 & 0.797 \\
\hline 330 & 5.512 & 0.988 & 0.741 & 1.87 \\
\hline 360 & 5.9349 & 1.145 & 0.229 & 1.515 \\
\hline \multicolumn{5}{|c|}{ (b) } \\
\hline \multicolumn{2}{|c|}{ Bounds on NP Parameter } & \multicolumn{3}{|c|}{ Branching Fraction $\left(\times 10^{-28}\right)$} \\
\hline$\theta$ & $\left|\lambda_{1 \mathrm{k} 3}^{\prime \star} \lambda_{1 \mathrm{k} 2}^{\prime}\right| \times 10^{-7}$ & NP & Interference & Combined \\
\hline 0 & 8.7538 & 2.56 & -1.216 & 1.489 \\
\hline 30 & 8.3927 & 2.36 & -0.183 & 2.322 \\
\hline
\end{tabular}


(b) Continued.

\begin{tabular}{lcccc}
\hline \multicolumn{3}{c}{ Bounds on NP Parameter } & \multicolumn{2}{c}{ Branching Fraction $\left(\times 10^{-28}\right)$} \\
$\theta$ & $\left|\lambda_{1 \mathrm{k} 3}^{\prime *} \lambda_{1 \mathrm{k} 2}^{\prime}\right| \times 10^{-7}$ & NP & Interference & Combined \\
\hline 60 & 7.4629 & 1.86 & 0.996 & 3.001 \\
90 & 6.3358 & 1.34 & 0.4 & 1.885 \\
120 & 5.3788 & 0.97 & -0.612 & 0.503 \\
150 & 4.7829 & 0.77 & -0.471 & 0.444 \\
180 & 4.5856 & 0.7 & 0.387 & 1.232 \\
210 & 4.7829 & 0.77 & 0.586 & 1.501 \\
240 & 5.3788 & 0.97 & -0.246 & 0.869 \\
270 & 6.3358 & 1.34 & -0.866 & 0.619 \\
300 & 7.4629 & 1.86 & 0.028 & 2.033 \\
330 & 8.3927 & 2.36 & 1.159 & 3.664 \\
360 & 8.7538 & 2.56 & 0.352 & 3.057 \\
\hline
\end{tabular}

(c)

\begin{tabular}{lcccc}
\hline \multicolumn{3}{c}{ Bounds on NP Parameter } & \multicolumn{3}{c}{ Branching Fraction $\left(\times 10^{-24}\right)$} & Combined \\
$\theta$ & $\left|\lambda_{2 \mathrm{k} 3}^{\prime *} \lambda_{2 \mathrm{k} 2}^{\prime}\right| \times 10^{-7}$ & $\mathrm{NP}$ & -0.91 & 1.111 \\
0 & 8.7538 & 1.913 & -0.134 & 1.732 \\
30 & 8.3927 & 1.758 & 0.74 & 2.238 \\
60 & 7.4629 & 1.39 & 0.296 & 1.406 \\
90 & 6.3358 & 1.002 & -0.455 & 0.375 \\
120 & 5.3788 & 0.722 & -0.347 & 0.332 \\
150 & 4.7829 & 0.571 & 0.286 & 0.919 \\
180 & 4.5856 & 0.525 & 0.44 & 1.119 \\
210 & 4.7829 & 0.571 & -0.182 & 0.648 \\
240 & 5.3788 & 0.722 & -0.648 & 0.462 \\
270 & 6.3358 & 1.002 & 0.018 & 1.516 \\
300 & 7.4629 & 1.39 & 0.867 & 2.733 \\
330 & 8.3927 & 1.758 & 0.259 & 2.28 \\
360 & 8.7538 & 1.913 & & \\
\hline
\end{tabular}

(d)

\begin{tabular}{lcccc}
\hline \multicolumn{3}{c}{ Bounds on NP Parameter } & \multicolumn{2}{c}{ Branching Fraction $\left(\times 10^{-22}\right)$} \\
$\theta$ & $\left|\lambda_{3 \mathrm{k} 3}^{\prime *} \lambda_{3 \mathrm{k} 2}^{\prime}\right| \times 10^{-7}$ & NP & Interference & Combined \\
\hline 0 & 8.7539 & 2.472 & -1.177 & 2.239 \\
30 & 8.3927 & 2.273 & -0.174 & 2.893 \\
60 & 7.4629 & 1.797 & 0.956 & 0.382 \\
90 & 6.3357 & 1.295 & -0.588 & 0.485 \\
120 & 5.3788 & 0.933 & -0.45 & 0.428 \\
150 & 4.7829 & 0.738 & 0.37 & 1.188 \\
180 & 4.5856 & 0.678 & 0.569 & 1.447 \\
210 & 4.7829 & 0.738 & -0.235 \\
240 & 5.3788 & 0.933 & -0.838 \\
270 & 6.3357 & 1.295 & 0.022 \\
300 & 7.4629 & 1.797 & 1.119 \\
330 & 8.3927 & 2.273 & 0.335 \\
360 & 8.7539 & 2.472 & 0.597 \\
\hline
\end{tabular}




\section{References}

[1] S. L. Glashow, J. Iliopoulos, and L. Maiani, "Weak interactions with lepton-hadron symmetry," Physical Review D: Particles, Fields, Gravitation and Cosmology, vol. 2, no. 7, pp. 1285-1292, 1970.

[2] Y. Fukuda, T. Hayakawa, E. Ichihara, (Super-Kamiokande Collaboration) et al., "Evidence for oscillation of atmospheric neutrinos," Physical Review Letters, vol. 81, article 1562, 1998.

[3] G. Mitsuka, K. Abe, Y. Hayato et al., "Study of Non-Standard Neutrino Interactions with Atmospheric Neutrino Data in Super-Kamiokande I and II," Physical Review D, vol. 84, article 113008, 2011.

[4] LSND Collaboration, L. B. Auerbach, R. L. Burman, and D. O. Caldwell, "Measurement of electron-neutrino electron elastic scattering," Physical Review D, vol. D63, article 112001, 2001.

[5] NuTeV Collaboration, G. P. Zeller, K. S. McFarland, and T. Adams, "A Precise Determination of Electroweak Parameters in Neutrino-Nucleon Scattering," Physical Review Letters, vol. 88, article 091802, 2002.

[6] J. A. B. Coelho and (MINOS Collaboration), "Search for effects of exotic models in MINOS Poster," in Proceedings of the 25th International Conference on Neutrino Physics and Astrophysics (Neutrino 2012), Kyoto, Japan, June 2012.

[7] Q. R. Ahmad, (SNO Collaboration) et al., "Measurement of the Rate of $v \mathrm{e}+\mathrm{d} \longrightarrow \mathrm{p}+\mathrm{p}+\mathrm{e}-$ Interactions Produced by $8 \mathrm{~B}$ Solar Neutrinos at the Sudbury Neutrino Observatory," Physical Review Letters, vol. 87, article 071301, 2001.

[8] K. Eguchi, (KamLAND Collaboration) et al., "First Results from KamLAND: Evidence for Reactor Anti-Neutrino Disappearance," Physics Review Letters, vol. 90, article 021802, Article ID 021802, 2003.

[9] Z. Isvan and (MINOS Collaboration), "Search for Non-standard Interactions with the MINOS Experiment," https://arxiv.org/ abs/1110.1900vl.

[10] F. P. An, J. Z. Bai, A. B. Balantekin et al., "Observation of electron-antineutrino disappearance at Daya Bay," Physical Review Letters, vol. 108, article 171803, Article ID 171803, 2012.

[11] Y. Abe, C. Aberle, T. Akiri et al., "Indication for the disappearance of reactor electron antineutrinos in the Double Chooz experiment," Physical Review Letters, vol. 108, article 131801, 2012.

[12] Y. Abe, C. Aberle, J. C. dos Anjos, (Double Chooz Collaboration) et al., "Reactor electron antineutrino disappearance in the Double Chooz experiment," Physical Review D, vol. 86, article 052008, 2012.

[13] J. K. Ahn, (RENO Collaboration) et al., "Observation of Reactor Electron Antineutrino Disappearance in the RENO Experiment," Physical Review Letters, vol. 108, article 191802, 2012.

[14] The Belle Collaboration, H. Tajima et al., "Belle B Physics Results," International Journal of Modern Physics A, vol. 2967, no. A17, 2002.

[15] R. Mizuk, R. Chistov et al., "Observation of two resonancelike structures in the $\pi+\chi \mathrm{cl}$ mass distribution in exclusive B0 $\longrightarrow \mathrm{K}-\pi+\chi \mathrm{cl}$ decays," Physical Review D, vol. 78, 072004, 2008.

[16] B. Aubert and (BABAR Collaboration), "Observation of the Semileptonic Decays $\mathrm{B} \longrightarrow \mathrm{D} * \tau-\nu \tau$ and Evidence for $\mathrm{B} \longrightarrow \mathrm{D} \tau-$ $v \tau$," Physical Review Letters, vol. 100, article 021801, 2008.

[17] A. Paul, I. I. Bigi, and S. Recksiegel, "D0 $\longrightarrow \gamma \gamma$ and D0 $\longrightarrow \mu+\mu-$ rates on an unlikely impact of the littlest Higgs model with
T parity," Physical Review D: Particles, Fields, Gravitation and Cosmology, vol. 83, no. 1, 2011.

[18] Y. Aida, E. Asakawa, G. Cho, and H. Matsuo, "Leptonic decays of Ds and $\mathrm{B}+$ mesons in the supersymmetric standard model with R-parity violating interactions," Physical Review D: Particles, Fields, Gravitation and Cosmology, vol. 82, no. 11, 2010.

[19] G. Bhattacharyya, K. Brata Chatterjee, and S. Nandi, "Correlated enhancements in Ds $\longrightarrow \ell v$, (g-2) of muon, and lepton flavor violating $\tau$ decays with two R-parity violating couplings," Nuclear Physics B, vol. 831, article 344, 2010.

[20] B. Bhattacherjee, G. Bhattacharyya, and S. Raychaudhuri, "Can Flavor Physics Hint at Distinctive Signals for R-parity Violation at the LHC?" Physical Review D, vol. 84, 075006, 2011.

[21] J. H. Jang, Y. G. Kim, and J. S. Lee, "BXsl+il-j decays with Rparity violation," Physical Review D, vol. 58, article 035006, 1998.

[22] Z. Xiao and L. Guo, "B0-B0 mixing and $\longrightarrow$ BXs $\gamma$ decay in the third type 2HDM: Effects of NLO QCD contributions," Physical Review D, vol. 69, article 014002, 2004.

[23] C.-S. Huang, "Neutral Higgs boson contributions to rare leptonic and semi-leptonic B decays in 2HDM and MSSM," Frontiers of Physics in China, vol. 2, pp. 212-233, 2007.

[24] J. P. Idarraga, R. Martinez, N. Poveda, and J. A. Rodriguez, "B -> X_s $\gamma$ and B $+->1+v$ in the 2HDM type III," https://arxiv.org/abs/ hep-ph/0509072.

[25] R. Ferrandes, "Probing Universal Extra Dimensions through rare decays induced by $\mathrm{b} \longrightarrow \mathrm{s}$ transition," in Proceedings of the AIP Conference, vol. 964, article 96, 2007.

[26] Y. Xu, R. Wang, and Y. Yang, "Probe R-parity violating supersymmetry effects in $\mathrm{B} \longrightarrow \mathrm{K}\left({ }^{*}\right) \ell+\ell-$ and $\mathrm{Bs} \longrightarrow \ell+\ell-$ decays," Physical Review D: Particles, Fields, Gravitation and Cosmology, vol. 74, no. 11, 2006.

[27] F. De Fazio, Minneapolis 2006, Continuous advances in QCD, 2006.

[28] C. S. Kim and R. Wang, "Study of the exclusive ", Physical Review D: Particles, Fields, Gravitation and Cosmology, vol. 77, no. 9, 2008.

[29] F. Domingo and U. Ellwanger, "Updated constraints from B physics on the MSSM and the NMSSM," Journal of High Energy Physics, vol. 2007, no. 12, pp. 090-090, 2007.

[30] S. Trine, "The MSSM Higgs sector and B-Bbar mixing for large $\tan$ (beta)," https://arxiv.org/abs/0710.4955.

[31] Y. Yamada, " $\mathrm{b} \longrightarrow \mathrm{s} \nu \boldsymbol{v}$ decay in the MSSM: Implication of $\mathrm{b} \longrightarrow \mathrm{s} \gamma$ at large $\tan \beta$," Physical Review D, vol. 77, article 014025, 2008.

[32] C. Bobeth, G. Hiller, and G. Piranishvili, "Angular distributions of B $\longrightarrow$ Kll decays," Journal of High Energy Physics, vol. 2007, no. 12, article no. 040, 2007.

[33] I. Ahmed, M. Paracha, and M. Aslam, "Exclusive B $\longrightarrow \mathrm{K} 1 \ell+\ell$ decay in model with single universal extra dimension," The European Physical Journal C, vol. 54, no. 4, pp. 591-599, 2008.

[34] D. G. Hitlin (Caltech), in Proceedings of the AIP Conference, vol. 870, article 398-404, 2006.

[35] A. Palano, Nuclear Physics B - Proceedings Supplements, 156, $105,2006$.

[36] A. Romano, "The $\mathrm{K}+\longrightarrow \pi+\nu v$ decay in the NA62 experiment at CERN," https://arxiv.org/abs/1411.6546.

[37] G. D’Ambrosio and A. M. Iyer, "Flavour issues in warped custodial models: B anomalies and rare $\mathrm{K}$ decays," https://arxiv.org/abs/1712.08122.

[38] T. K. Komatsubara and Prog. Part, "Experiments with K-Meson Decays," Progress in Particle and Nuclear Physics, vol. 67, article 995, 2012. 
[39] KOTO Collaboration and K. Shiomi, " $\mathrm{K}_{0}^{L} \longrightarrow \pi 0 \nu v$ at KOTO," in Proceedings of the 8th International Workshop on the CKM Unitarity Triangle (CKM 2014), Vienna, Austria, 2014.

[40] K. F. Chen et al., "B-Physics at CMS with LHC Run-II and Beyond," Proceedings of Science, vol. 549, 2015.

[41] Luca. Martini, "The CMS observation of the rare decay B s 0 $\longrightarrow \mu+\mu$ - with the LHC Run I data," The European Physical Journal Plus, vol. 130, article 184, 2015.

[42] H. E. Haber and G. L. Kane, "The search for supersymmetry: probing physics beyond the standard model," Physics Reports, vol. 117, pp. 75-263, 1985.

[43] R. Haag, J. T. Lopuzanski, and M. F. Sohnius, "All possible generators of supersymmetries of the S-matrix," Nuclear Physics $B$, vol. 88, no. 2, pp. 257-274, 1975.

[44] P. Fayet, Karpacz Winter School (1980) 115 (QC174.45:W5:1980).

[45] S. P. Martin, "A Supersymmetry Primer," Advanced Series on Directions in High Energy Physics, vol. 18, pp. 1-98, 1998.

[46] R. Barbier, C. Bérat, and M. Besançon, " $R$-parity-violating supersymmetry," Physics Reports, vol. 420, pp. 1-202, 2005.

[47] H. K. Dreiner, M. Kramer, and B. O’Leary, "Bounds on Rparity violating supersymmetric couplings from leptonic and semi-leptonic meson decays," Physical Review D, vol. 75, article 114016, 2007.

[48] R. Haag, J. T. Lopuszanski, and M. Sohnius, "All possible generators of supersymmetries of the S-matrix," Nuclear Physics $B$, vol. 88, pp. 257-274, 1975.

[49] W. Buchmüller and D. Wyler, " $C P$ violation and $R$ invariance in supersymmetric models of strong and electroweak interactions," Physics Letters B, vol. 121, no. 5, pp. 321-325, 1983.

[50] F. Tahir, M. A. Mughal, and K. Ahmed, "Europhysics Letters 54," pp. 580-585, 2001.

[51] A. Mir, F. Tahir, M. Q. Haseeb, and K. Ahmed, "Implications of $R$ parity violating Yukawa couplings in $\Delta S=1$ semileptonic decays of $K$ mesons," Physical Review D: Particles, Fields, Gravitation and Cosmology, vol. 76, no. 11, 2007.

[52] A. Mir, F. Tahir, and K. Ahmed, "epton polarization asymmetry in $\mathrm{B} \longrightarrow \mathrm{l}+\mathrm{l}$ - decays in R-parity-violating minimal supersymmetric standard model," Europhysics Letters, vol. 83, no. 4, 2008.

[53] T. Inami and C. S. Lim, "Effects of superheavy quarks and leptons in low-energy weak processes $K_{L} \longrightarrow \mu \bar{\mu}, K^{+} \longrightarrow \pi^{+} \nu \bar{v}$ and $K^{0} \longleftrightarrow \bar{K}^{0}$, , Progress of Theoretical and Experimental Physics, vol. 65, p. 287, 1981.

[54] N. Cabibbo, "Unitary symmetry and leptonic decays," Physical Review Letters, vol. 10, no. 12, pp. 531-533, 1963.

[55] M. Kobayashi and K. Maskawa, "CP Violation in the Renormalizable Theory of Weak Interaction," Progress of Theoretical Physics, vol. 49, pp. 652-657, 1973.

[56] D. Rein and L. M. Sehgal, "Long-distance contributions to the decay ," Physical Review D: Particles, Fields, Gravitation and Cosmology, vol. 39, no. 11, pp. 3325-3329, 1989.

[57] F. Tahir, M. Anwar Mughal, and K. Ahmed, "New bounds on the products of R-parity breaking couplings from two-body pseudoscalar mesonic decays," Europhysics Letters, vol. 54, no. $5,2001$.

[58] K. Lee and P. Yi, "Monopoles and instantons on partially compactified D-branes," Physical Review D: Particles, Fields, Gravitation and Cosmology, vol. 56, no. 6, pp. 3711-3717, 1997.

[59] J. Jang, J. K. Kim, and J. S. Lee, “ Constraints on the ” Physical Review D: Particles, Fields, Gravitation and Cosmology, vol. 55, no. 11, pp. 7296-7299, 1997.
[60] J. Jang, Y. G. Kim, and J. S. Lee, "R-parity violation and semileptonic decays of B-meson," Physics Letters B, vol. 408, no. 1-4, pp. 367-372, 1997.

[61] D. Choudhury and P. Roy, "New constraints on lepton nonconserving R-parity violating couplings," Physics Letters B, vol. 378, no. 1-4, pp. 153-158, 1996.

[62] A. J. Buras, "Weak Hamiltonian, CP violation and rare decays," https://arxiv.org/abs/hep-ph/9806471.

[63] A. J. Buras, D. Buttazzo, J. Girrbach-Noe, and R. Knegjens,

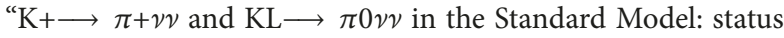
and perspectives," Journal of High Energy Physics, vol. 33, 2015.

[64] J. Jang, Y. G. Kim, and J. S. Lee, " $\mathrm{BX}_{s} l_{i}^{+} 1_{j}^{-}$decays with R-parity violation," Physical Review D: Particles, Fields, Gravitation and Cosmology, vol. 58, no. 3, 1998.

[65] W. J. Marciano and Z. Parsa, "Rare kaon decays with "missing energy"', Physical Review D: Particles, Fields, Gravitation and Cosmology, vol. 53, no. 1, pp. R1-R5, 1996.

[66] J. L. Rosner, S. Stone, and R. S. Van de, "Leptonic Decays of Charged Pseudoscalar Mesons - 2015," https://arxiv.org/abs/ 1509.02220.

[67] W. Wang and Z. Xiao, "Semileptonic decays " Physical Review D: Particles, Fields, Gravitation and Cosmology, vol. 86, no. 11, 2012.

[68] C. Patrignani, (Particle Data Group) et al., "Review of Particle Physics," Chinese Physics C, vol. 40, no. 10, Article ID 100001, 2016. 

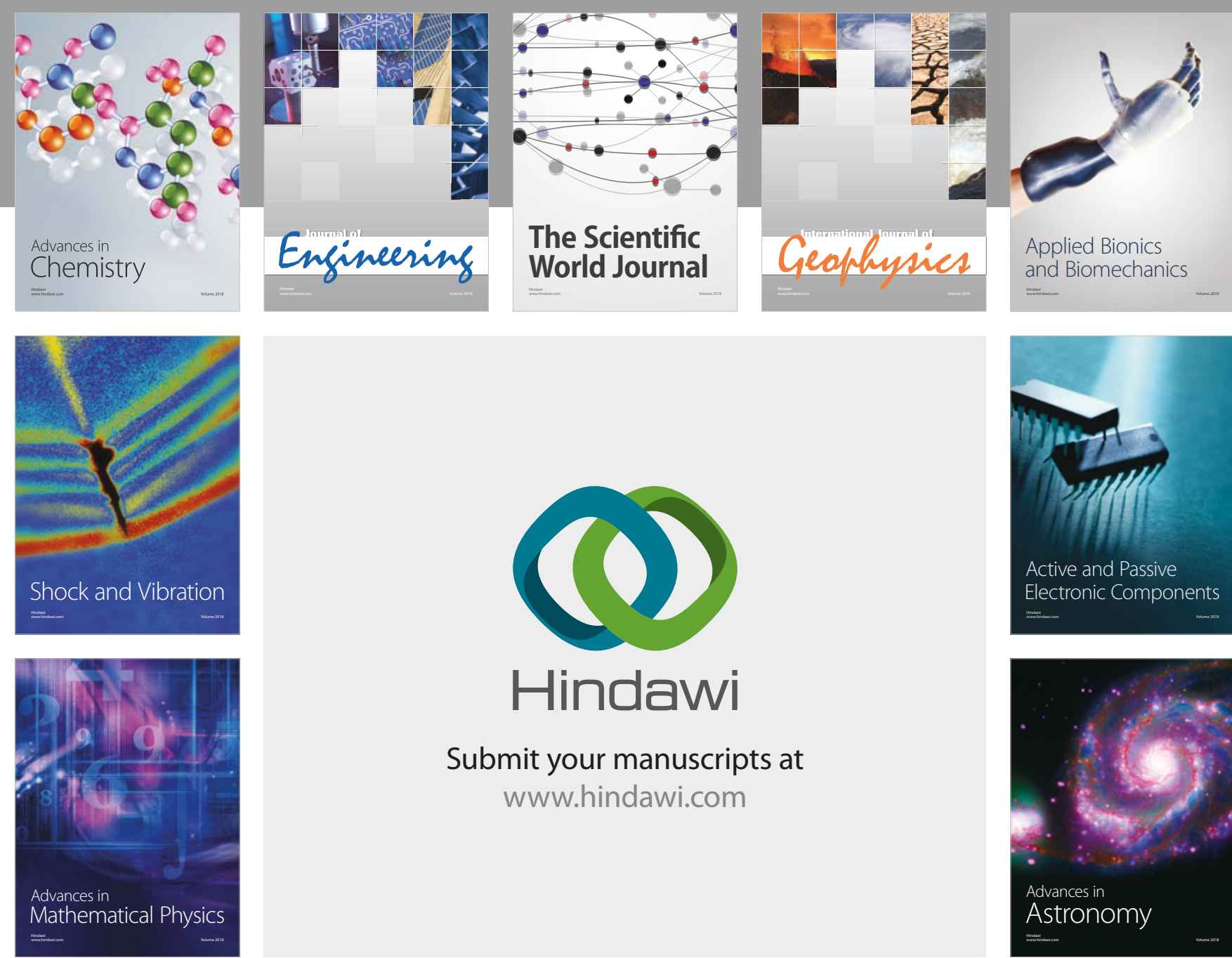

Submit your manuscripts at

www.hindawi.com

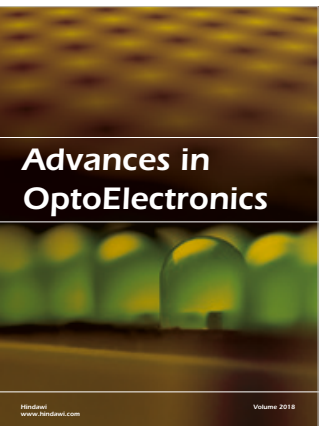

\section{Rotcting Machinery}
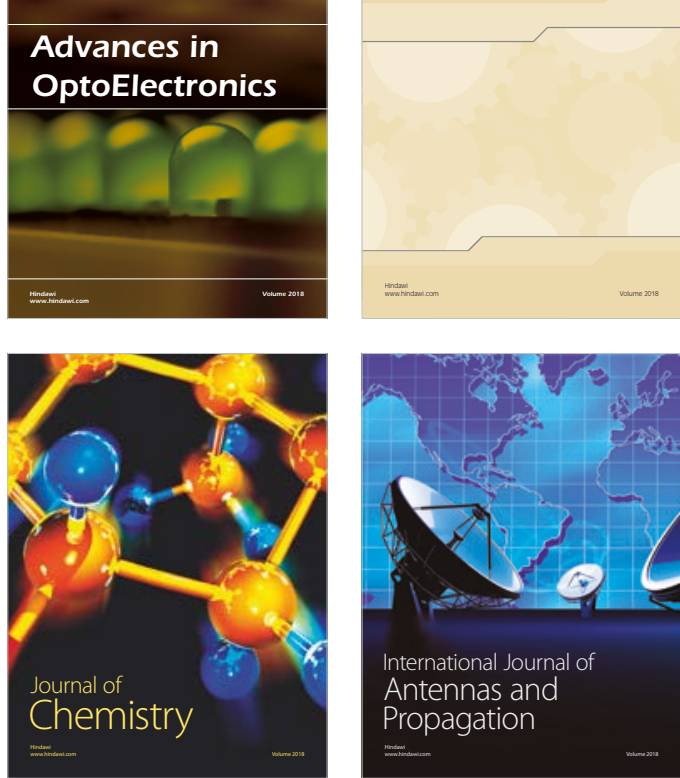

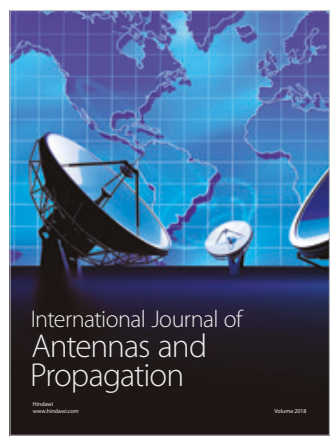

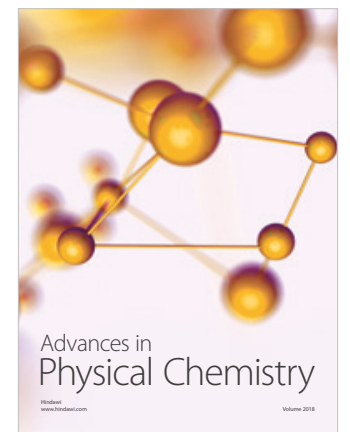

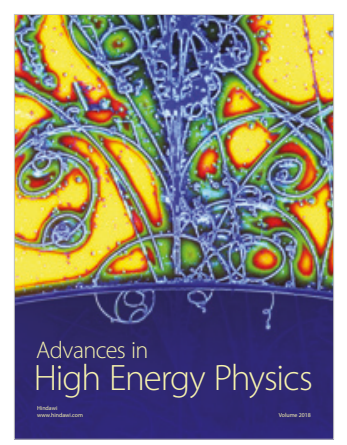

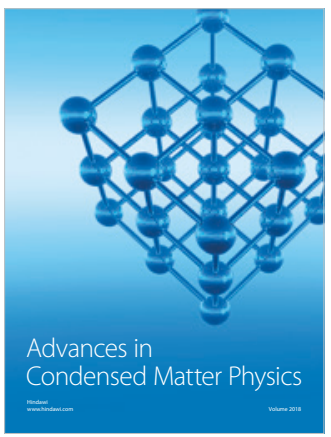

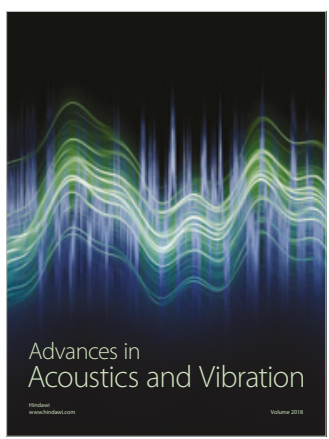

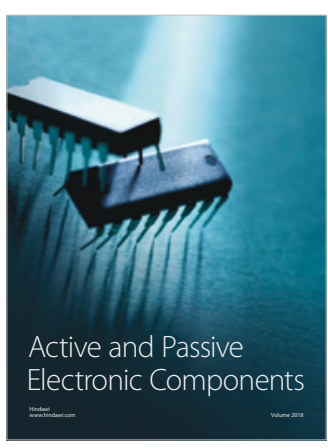
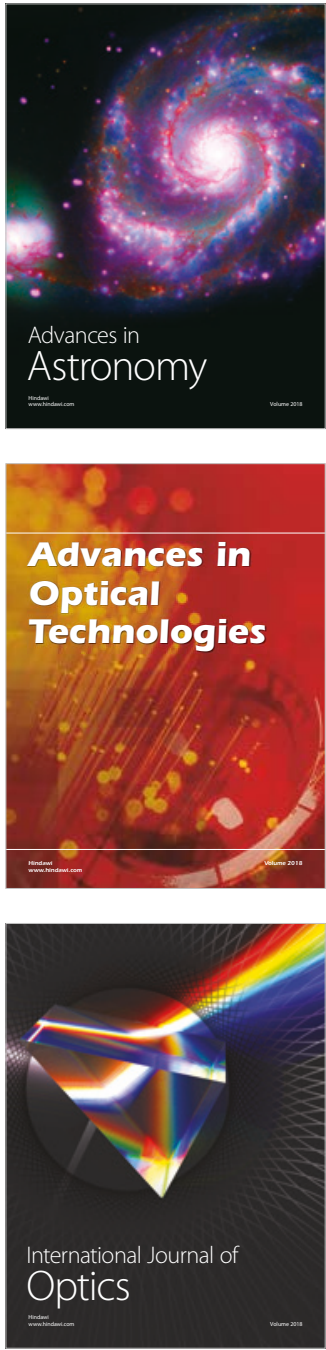